$\begin{array}{ll}\text { Research Square } & \begin{array}{l}\text { Preprints are preliminary reports that have not undergone peer review. } \\ \text { They should not be considered conclusive, used to inform clinical practice, } \\ \text { or referenced by the media as validated information. }\end{array}\end{array}$

\title{
The Sex Difference and Potential Influence Factors In 96,237 Patients With Coronavirus Disease 2019 (COVID-19): A Systematic Review and Meta- Analysis
}

\author{
Lingwei Ma \\ Tongji Hospital of Tongji Medical College of Huazhong University of Science and Technology \\ Huan Lu \\ Tongji Hospital of Tongji Medical College of Huazhong University of Science and Technology \\ Meng Wu \\ Tongji Hospital of Tongji Medical College of Huazhong University of Science and Technology \\ Liru Xue \\ Tongji Hospital of Tongji Medical College of Huazhong University of Science and Technology \\ QingQing Zhu \\ Tongji Hospital of Tongji Medical College of Huazhong University of Science and Technology \\ Jinjin Zhang \\ Tongji Hospital of Tongji Medical College of Huazhong University of Science and Technology \\ Shixuan Wang ( $\square$ shixuanwang@tjh.tjmu.edu.cn ) \\ Tongji Hospital of Tongji Medical College of Huazhong University of Science and Technology
}

Research article

Keywords: COVID-19, SARS-CoV-2 (2019-nCoV), sex difference, meta-analysis, sex hormone, immune response, social behavior

Posted Date: December 22nd, 2020

DOI: https://doi.org/10.21203/rs.3.rs-132290/v1

License: (9) (7) This work is licensed under a Creative Commons Attribution 4.0 International License. Read Full License 


\section{Abstract}

Background: Coronavirus disease 2019 (COVID-19) is an infectious disease caused by severe acute respiratory syndrome coronavirus 2 (SARS-CoV-2). This disease was first identified in December 2019 in Wuhan and has since spread globally, resulting in the ongoing 2019-20 coronavirus pandemic. Numerous studies of the clinical characteristics of patients with SARS-CoV-2 have been reported, yet yielded varying results given the sex difference of prevalence, severity and fatality rate. We conducted a review and meta-analysis of the sex differences of COVID-19, aiming to reveal the epidemiological characteristics and potential influencing factors of COVID-19 and provide reference for the prevention and treatment of patients.

Results: By screening 1436 studies, 156 studies including 96237 COVID-19 patients from multiple countries were included. We found out that females account for 0.47 [95\% $\mathrm{Cl}(0.45-0.48)]$ of the diagnosed COVID-19 cases, while males for 0.53 of the cases $[95 \% \mathrm{Cl}(0.52-0.55)]$. Females admitted into intensive care unit (ICU) or with severe/critical illness were also analyzed, and the ratio was 0.14 [95\% $\mathrm{Cl}(0.11-0.17)]$ among all the severely/critically ill patients, which was almost twice lower than the ratio of males $(0.24$ [95\% $\mathrm{Cl}(0.19-0.29)])$. The fatality rate of female patients was 0.10 [95\% $\mathrm{Cl}(0.05-0.17)]$, which was much lower than that of males $(0.18[95 \% \mathrm{Cl}(0.11-0.29)])$.

Conclusions: There exists a significantly different clinical outcome between male and female COVID-19 patients. Females are less severely affected, which might be associated with the sex-based difference in immune response, female hormones and behaviors. However, more specific studies are needed to identify more relevant factors.

\section{Background}

Since December 2019, the current outbreak of coronavirus disease (COVID-19) caused by a novel coronavirus (SARS-CoV-2, previously named 2019-nCoV) was first identified in Wuhan, Hubei Province of the People's Republic of China [1] and aroused attention of worldwide. On March 11, 2020, The World Health Organization (WHO) officially announced this rapidly spreading coronavirus outbreak as a pandemic based on the fact that the number of confirmed cases of COVID-19 outside China has increased rapidly and dramatically.

The next-generation sequencing and comparative homology analysis revealed that this novel pathogen belongs to a member of lineage B within genus Betacoronavirus with a significantly different genomic sequence from SARS-CoV (about 79\% identity) and MERS-CoV (about 50\% identity)[2].

The clinical spectrum of COVID-19 is varied, ranging from asymptomatic form or non-specific signs to acute respiratory distress syndrome (ARDS) and systemic manifestations of sepsis and septic shock, i.e. multiple organ dysfunction syndromes (MODS).

At present there is an emerging collection of the basic research, clinical and epidemiological data about COVID-19.The initial report of 41 COVID-19 patients in Wuhan demonstrated that more men (73\%) patients were affected than were women [3]. According to Guan et al.'s data [3] on the clinical characteristics of 1099 cases in 30 provinces in China, male patients accounted for 58\%. Moreover, 35 (67\%) of critically ill patients admitted to the intensive care unit (ICU) were male patients [4]. Based on the public database and the analysis of single-cell RNA expression profiles of human normal lung cells, it was found that the expression level of Angiotensin-converting enzyme 2 (ACE2, considered as an SARS-CoV-2 receptor [5]) in Asian men is higher (preprint)[6]. Thus, it is speculated that there might be differences in susceptibility to COVID-19 between two sexes, and males are more prone to suffering from severe clinical conditions than are females. However, these reports are limited with a small cohort of cases.

\section{Methods Objective}

In order to make a more convincing conclusion, we have conducted a systematic evaluation of the sex predisposition of COVID-19 by analyzing a large cohort of reported clinical studies and cases globally. In our research, the clinical data between December, 2019 and April, 2020 were extracted and collected to discover and reveal the difference of incidence, first symptoms, severity, and outcomes between two sexes. Clarifying the sex difference in susceptibility and severity of COVID-19 and identifying possible risky factors can help protect more vulnerable populations and provide useful information for developing better treatment and prognosis of COVID-19.

\section{Eligibility criteria}

Studies were included if they reported confirmed patients with COVID-19, and reported the sex distribution of patients. Repeated cases, reviews, editorials, family or cluster-based studies, only children (including neonates) cases and only pregnant women cases were excluded.

\section{Information sources}

A comprehensive systematic literature search of online databases was conducted on April 27, 2020, including Pubmed, Embase, Web of Science, medRxiv, bioRxiv and Arxiv to collect all case studies. The EndNote X9.0 software was used to manage and exclude the duplicated literatures.

\section{Search strategy}

The search terms were as follows: "SARS-CoV-2", "COVID-19", "2019 novel coronavirus", "2019-nCoV", "clinical characteristics", "clinical features" and "clinical manifestations" without any language restriction. The database search results were conducted according to the flow of Preferred Reporting for Systematic Reviews of Meta-analyses (PRISMA) statement[7].

\section{Data extraction and analysis}


All available publications have been carefully analyzed and strictly reviewed by two reviewers. Microsoft Excel was used to record all available information, including the authors, source place, collection period, age, case load, sex, the number of patients in ICU-male, ICU-female, the prevalence of mild/common illness and severe/critical illness, discharge rate, remained in hospital rate and fatality rate. Any disagreements were resolved by discussion.

\section{Quality assessment and risk of bias}

An 11-item checklist for cross-sectional/prevalence study quality provided by Agency for Healthcare Research and Quality (AHRQ) [8] was applied to assess the quality of included studies. When answering 'NO' or 'UNCLAER', the item would be scored ' 0 '; When answering 'YES', the item would be scored ' 1 '. The included studies were rated with following scores: low quality (0-3); moderate quality (4-7); high quality (8-11). Publication bias was examined by using a funnel-plot with Begg's and Egger's test.

\section{Data analysis}

The statistical analyses were performed by using R (version 3.6.2) and STATA MP (version 13.0). As there exists heterogeneity between and within studies, a random effect model was used to calculate the pooled prevalence and $95 \% \mathrm{Cl}$, which was demonstrated by forest plot. Heterogeneity among studies was tested using the Cochran's Q test and $\mathrm{I}^{2}$ (ranged from $0-100 \%$ ). When $\mathrm{I}^{2}<50 \%$, fixed-effects model was used, while when $\mathrm{I}^{2}>50 \%$, random-effects was selected. The R package "meta" was used to analyze and display plots. Funnel plots were used to assess the publication bias. $P<0.05$ was considered as statistical significance (2-sided).

\section{Results}

A total of 1436 literatures were searched from the online databases based on the strategy. After we removed duplicated studies, screened the title and abstract and reviewed full-text, 156 studies were selected in the meta-analysis. Data was extracted from published manuscripts. The flowchart of the selection process was displayed in Fig. 1. The quality score of each study was displayed in Table 1. 8 studies were of high quality and 148 were of moderate quality. No articles were of low-quality rating. 
Table 1

Main Characteristics of included studies in this meta-analysis

\begin{tabular}{|c|c|c|c|c|c|c|c|c|c|}
\hline Study & $\begin{array}{l}\text { collection } \\
\text { period }\end{array}$ & Age & $\begin{array}{l}\text { Case } \\
\text { load }\end{array}$ & $\begin{array}{l}\text { Male } \\
\text { (n) }\end{array}$ & $\begin{array}{l}\text { ICU- } \\
\text { Male }\end{array}$ & $\begin{array}{l}\text { ICU- } \\
\text { Female }\end{array}$ & mild & common & severe \\
\hline $\begin{array}{l}\text { Zhu Z, et al. } \\
\text { (Wuhan) }\end{array}$ & $\begin{array}{l}\text { Jan } 23 \text { to } \\
\text { Feb 20, } \\
2020\end{array}$ & 50.90(15.26)_mean(SD) & 127 & 45 & / & / & 111_M73_F38 & & 16_M9_F' \\
\hline $\begin{array}{l}\text { Zhou Y, et al. } \\
\text { (Huangshi) }\end{array}$ & $\begin{array}{l}\text { Jan } 28 \text { to } \\
\text { Mar 2, } \\
2020\end{array}$ & 66.10(13.94)_mean(SD) & 21 & 13 & 13 & 8 & / & / & 8_M3_F5 \\
\hline $\begin{array}{l}\text { Zhou B, et al. } \\
\text { (Wuhan) }\end{array}$ & $\begin{array}{l}\text { Feb } 5 \text { to } \\
\text { Feb 13, } \\
2020\end{array}$ & / & 34 & 17 & / & / & / & / & 26_M12_F \\
\hline $\begin{array}{l}\text { Zheng Y, et al. } \\
\text { (Chengdu) }\end{array}$ & $\begin{array}{l}\text { Jan } 16 \text { to } \\
\text { Feb 20, } \\
2020\end{array}$ & 49.4(18.45)_mean(SD) & 99 & 51 & / & / & 67 & & / \\
\hline $\begin{array}{l}\text { Zheng S, et al. } \\
\text { (Zhejiang Province) }\end{array}$ & $\begin{array}{l}\text { Jan } 19 \text { to } \\
\text { Mar 20, } \\
2020\end{array}$ & $\begin{array}{l}\text { 55(44.3- } \\
\text { 46.8)_median(IQR) }\end{array}$ & 96 & 58 & 30 & & 22_M9_F13 & / & 74_M49_F \\
\hline $\begin{array}{l}\text { Zhao W, et al. } \\
\text { (Hunan Province) }\end{array}$ & / & $\begin{array}{l}43(17- \\
75) \_ \text {median(range) }\end{array}$ & 101 & 56 & / & I & 87_M48_F39 & l & 14_M8_Ft \\
\hline $\begin{array}{l}\text { Zhao W, et al. } \\
\text { (Hunan Province) }\end{array}$ & $\begin{array}{l}\text { Jan } 16 \text { to } \\
\text { Feb 4, } \\
2020\end{array}$ & 44.06(13.62)_mean(SD) & 118 & 60 & / & / & 8 & 100 & 10 \\
\hline $\begin{array}{l}\text { Zhang Y, et al. } \\
\text { (Wuhan) }\end{array}$ & $\begin{array}{l}\text { Jan } 18 \text { to } \\
\text { Feb 22, } \\
2020\end{array}$ & $\begin{array}{l}49.52 \\
(17.06) \_m e a n(S D)\end{array}$ & 115 & 49 & / & / & 84_M29_55 & / & 31_M20_F \\
\hline $\begin{array}{l}\text { Zhang X, et al. } \\
\text { (Zhejiang Province) }\end{array}$ & $\begin{array}{l}\text { Jan } 17 \text { to } \\
\text { Feb } 8 \\
2020\end{array}$ & 46.65(13.82)_mean(SD) & 573 & 295 & / & / & / & / & 64 \\
\hline $\begin{array}{l}\text { Zhang R, et al. } \\
\text { (Wuhan) }\end{array}$ & $\begin{array}{l}\text { Jan } 10 \text { to } \\
\text { Feb } 10, \\
2020\end{array}$ & 45.4(15.6)_mean(SD) & 120 & 43 & l & l & / & 90_M30_F60 & 30_M13_F \\
\hline $\begin{array}{l}\text { Zhang J, et al. } \\
\text { (Wuhan) }\end{array}$ & $\begin{array}{l}\text { Jan } 11 \text { to } \\
\text { Feb 6, } \\
2020\end{array}$ & $\begin{array}{l}\text { 55.6(44- } \\
69) \text { mean(IQR) }\end{array}$ & 663 & 321 & / & / & 254_M116_F138 & & 315_M14! \\
\hline $\begin{array}{l}\text { Zhang J, et al. } \\
\text { (Wuhan) }\end{array}$ & $\begin{array}{l}\text { Jan } 6 \text { to } \\
\text { Feb 20, } \\
2020\end{array}$ & $\begin{array}{l}73 \text { (38- } \\
91) \_ \text {median(range) }\end{array}$ & 19 & 11 & 11 & 8 & / & / & / \\
\hline $\begin{array}{l}\text { Zhang G, et al. } \\
\text { (Wuhan) }\end{array}$ & $\begin{array}{l}\text { Jan } 2 \text { to } \\
\text { Feb 10, } \\
2020\end{array}$ & $55.0(39.0-66.5)_{-}$ & 221 & 108 & 44 & & 166_M73_F93 & & 55_M35_F \\
\hline $\begin{array}{l}\text { Yuan M, et al. } \\
\text { (Wuhan) }\end{array}$ & $\begin{array}{l}\text { Jan } 1 \text { to } \\
\text { Jan } 25 \\
2020\end{array}$ & $\begin{array}{l}\text { 60(47- } \\
69) \text { median(IQR) }\end{array}$ & 27 & 12 & / & / & / & / & / \\
\hline $\begin{array}{l}\text { Young BE, et al. } \\
\text { (Singapore) }\end{array}$ & $\begin{array}{l}\text { Jan } 23 \text { to } \\
\text { Feb 3, } \\
2020\end{array}$ & $\begin{array}{l}\text { 47(31- } \\
\text { 73)_median(range) }\end{array}$ & 18 & 9 & / & / & / & / & / \\
\hline $\begin{array}{l}\text { Yao Q, et al. } \\
\text { (Huanggang, Hubei } \\
\text { Province) }\end{array}$ & $\begin{array}{l}\text { Jan } 30 \text { to } \\
\text { Feb 11, } \\
2020\end{array}$ & $\begin{array}{l}\text { 52(37- } \\
\text { 58)_median(IQR) }\end{array}$ & 108 & 43 & 17 & & / & 83_M30_F53 & 25_M13_F \\
\hline $\begin{array}{l}\text { Yang F, et al. } \\
\text { (Wuhan) }\end{array}$ & $\begin{array}{l}\text { Jan } 6 \text { to } \\
\text { Feb 25, } \\
2020\end{array}$ & 69.8 (14.5)_mean(SD) & 92 & 49 & / & / & / & / & / \\
\hline $\begin{array}{l}\text { Yang A-P, et al. } \\
\text { (Zhejiang Province) }\end{array}$ & $\begin{array}{l}\text { until Feb } \\
20,2020\end{array}$ & 46.4(17.6)_mean(SD) & 93 & 56 & / & / & 69_M38_F31 & & 24_M18_F \\
\hline $\begin{array}{l}\text { Xu Y-H, et al. } \\
\text { (Beijing) }\end{array}$ & $\begin{array}{l}\text { Jan-to } \\
\text { Feb, } 2020\end{array}$ & 43.9(16.8)_mean(SD) & 50 & 29 & / & / & 9_M7_F2 & 28_M15_F13 & 10_M7_F \\
\hline Xu B, et al.(Wuhan) & $\begin{array}{l}\text { Dec } 26 \text { to } \\
\text { Mar 1, } \\
2020\end{array}$ & $\begin{array}{l}\text { 62(48.5-71)_median } \\
\text { (IQR) }\end{array}$ & 187 & 103 & / & / & 80 & / & 45 \\
\hline $\begin{array}{l}\text { Xiong Y, et al. } \\
\text { (Wuhan) }\end{array}$ & $\begin{array}{l}\text { Jan } 11 \text { to } \\
\text { Feb 5, } \\
2020\end{array}$ & 49.5(14.1)_mean(SD) & 42 & 25 & / & / & / & / & l \\
\hline
\end{tabular}




\begin{tabular}{|c|c|c|c|c|c|c|c|c|c|}
\hline Study & $\begin{array}{l}\text { collection } \\
\text { period }\end{array}$ & Age & $\begin{array}{l}\text { Case } \\
\text { load }\end{array}$ & $\begin{array}{l}\text { Male } \\
\text { (n) }\end{array}$ & $\begin{array}{l}\text { ICU- } \\
\text { Male }\end{array}$ & $\begin{array}{l}\text { ICU- } \\
\text { Female }\end{array}$ & mild & common & severe \\
\hline Xie H, et al.(Wuhan) & $\begin{array}{l}\text { Feb } 2 \text { to } \\
\text { Feb 23, } \\
2020\end{array}$ & $\begin{array}{l}\text { 60.0(48.0- } \\
66) \text { median(IQR) }\end{array}$ & 79 & 44 & / & / & / & 51_M26_F25 & 28_M18_F \\
\hline $\begin{array}{l}\text { Wang X, et al. } \\
\text { (Wuhan) }\end{array}$ & $\begin{array}{l}\text { Jan } 10 \text { to } \\
\text { Feb 24, } \\
2020\end{array}$ & $\begin{array}{l}56 \text { (32- } \\
48.5) \_m e d i a n(I Q R)\end{array}$ & 80 & 31 & / & / & / & / & / \\
\hline $\begin{array}{l}\text { Wang X, et al. } \\
\text { (Wuhan) }\end{array}$ & $\begin{array}{l}\text { Feb } 7 \text { to } \\
\text { Feb 12, } \\
2020\end{array}$ & $\begin{array}{l}\text { 50(39-58)_median } \\
\text { (IQR) }\end{array}$ & 1012 & 524 & / & / & 1012_M524_F488 & & / \\
\hline $\begin{array}{l}\text { Wang R, et al. } \\
\text { (Fuyang, Anhui } \\
\text { Province) }\end{array}$ & $\begin{array}{l}\text { Jan } 20 \text { to } \\
\text { Feb 9, } \\
2020\end{array}$ & 28.76(13.80)_mean(SD) & 125 & 71 & / & / & 100_M55_F45 & & 25_M16_F \\
\hline $\begin{array}{l}\text { Wang L, et al. } \\
\text { (Wuhan) }\end{array}$ & $\begin{array}{l}\text { Jan } 1 \text { to } \\
\text { Feb 6, } \\
2020\end{array}$ & 71(8)_mean(SD) & 339 & 166 & / & / & / & 100 & 159 \\
\hline $\begin{array}{l}\text { Wang J, et al. } \\
\text { (Zhejiang, Anhui } \\
\text { Province) }\end{array}$ & $\begin{array}{l}\text { Jan to } \\
\text { Feb, } 2020\end{array}$ & 52.1(18.07)_mean(SD) & 93 & 57 & l & / & 73_M43_F30 & & 20_M14_F \\
\hline $\begin{array}{l}\text { Wang G, et al. } \\
\text { (Zhejiang Province) }\end{array}$ & $\begin{array}{l}\text { Jan } 27 \text { to } \\
\text { Mar } 3 \\
2020\end{array}$ & 47.82(15.19)_mean(SD) & 889 & 438 & / & l & / & / & l \\
\hline $\begin{array}{l}\text { Sun C, et al. } \\
\text { (Nanyang, Henan } \\
\text { Province) }\end{array}$ & $\begin{array}{l}\text { Jan } 24 \text { to } \\
\text { Feb 16, } \\
2020\end{array}$ & 45(16)_mean(SD) & 150 & 67 & / & / & 6 & 105 & 28 \\
\hline $\begin{array}{l}\text { Simonnet A, et al. } \\
\text { (France) }\end{array}$ & $\begin{array}{l}\text { Feb } 27 \text { to } \\
\text { April } 5 \\
2020\end{array}$ & $\begin{array}{l}\text { 60(51- } \\
\text { 70)_median(IQR) }\end{array}$ & 124 & 90 & 90 & 34 & / & / & / \\
\hline $\begin{array}{l}\text { Richardson S, et al. } \\
\text { (New York City) }\end{array}$ & $\begin{array}{l}\text { Mar } 1 \text { to } \\
\text { April 4, } \\
2020\end{array}$ & $\begin{array}{l}\text { 63(52- } \\
\text { 75)_median(IQR) }\end{array}$ & 5700 & 3437 & 248 & 125 & / & / & / \\
\hline $\begin{array}{l}\text { Qian ZP, et al. } \\
\text { (Shanghai) }\end{array}$ & $\begin{array}{l}\text { Jan } 20 \text { to } \\
\text { Feb 24, } \\
2020\end{array}$ & $\begin{array}{l}\text { 51(15- } \\
\text { 88)_median(range) }\end{array}$ & 324 & 167 & / & / & 298_M147_F151 & & 26_M20_F \\
\hline Niu S, et al.(Beijing) & $\begin{array}{l}\text { Jan } 20 \text { to } \\
\text { Feb 29, } \\
2020\end{array}$ & / & 60 & 34 & / & / & / & / & / \\
\hline $\begin{array}{l}\text { Lyu P, et al. } \\
\text { (Zhengzhou) }\end{array}$ & $\begin{array}{l}\text { Jan } 15 \text { to } \\
\text { Feb 24, } \\
2020\end{array}$ & 54(17)_mean(SD) & 51 & 29 & 23 & & 12_M7_F5 & & 15_M8_F' \\
\hline Liu Y, et al.(Wuhan) & $\begin{array}{l}\text { Jan } 1 \text { to } \\
\text { Feb 29, } \\
2020\end{array}$ & 53.95(16.90)_mean(SD) & 245 & 114 & l & / & / & / & / \\
\hline $\begin{array}{l}\text { Liu K-C, et al.(Anhui } \\
\text { Province) }\end{array}$ & $\begin{array}{l}\text { Jan } 21 \text { to } \\
\text { Feb 3, } \\
2020\end{array}$ & 41.6(14.5)_mean(SD) & 73 & 41 & / & / & 6_M3F3 & 43_M28F15 & 21_M10F \\
\hline Liu K, et al.(Hainan) & $\begin{array}{l}\text { Jan } 15 \text { to } \\
\text { Feb } 18 \\
2020\end{array}$ & / & 56 & 31 & / & / & / & / & / \\
\hline $\begin{array}{l}\text { Liang W-H, et al. } \\
\text { (Mainland China) }\end{array}$ & / & 48.9(16.3)_mean(SD) & 1590 & 904 & 99 & & 907 & & 36 \\
\hline Li X, et al.(Wuhan) & $\begin{array}{l}\text { Jan } 26 \text { to } \\
\text { Feb 5, } \\
2020\end{array}$ & $\begin{array}{l}\text { 60(48- } \\
69) \text { median(IQR) }\end{array}$ & 548 & 279 & / & / & 279_M126F153 & & 269_M15: \\
\hline Li K, et al.(/) & $\begin{array}{l}\text { Jan to } \\
\text { Feb, } 2020\end{array}$ & 45.5(12.3)_mean(SD) & 83 & 44 & / & / & 58_M29F29 & & 25_M15F' \\
\hline $\begin{array}{l}\text { Lei Z, et al.(Wuhan, } \\
\text { Guangzhou) }\end{array}$ & $\begin{array}{l}\text { Jan } 22 \text { to } \\
\text { Feb12, } \\
2020 ; \text { Jan } \\
1 \text { to Feb } \\
20,2020\end{array}$ & / & 119 & 77 & / & / & / & / & / \\
\hline $\begin{array}{l}\text { He GH, et al. } \\
\text { (Guanghzou, } \\
\text { Wenzhou) }\end{array}$ & $\begin{array}{l}\text { By Feb } \\
29,2020\end{array}$ & / & 811 & 411 & / & / & / & / & I \\
\hline
\end{tabular}




\begin{tabular}{|c|c|c|c|c|c|c|c|c|c|}
\hline Study & $\begin{array}{l}\text { collection } \\
\text { period }\end{array}$ & Age & $\begin{array}{l}\text { Case } \\
\text { load }\end{array}$ & $\begin{array}{l}\text { Male } \\
(n)\end{array}$ & $\begin{array}{l}\text { ICU- } \\
\text { Male }\end{array}$ & $\begin{array}{l}\text { ICU- } \\
\text { Female }\end{array}$ & mild & common & severe \\
\hline $\begin{array}{l}\text { Han } \mathrm{C} \text {, et al. } \\
\text { (Wuhan) }\end{array}$ & $\begin{array}{l}\text { Feb } 13 \text { to } \\
\text { Feb 29, } \\
2020\end{array}$ & $\begin{array}{l}\text { 62.5(27- } \\
\text { 92)_median(IQR) }\end{array}$ & 206 & 91 & / & / & 206_M91_F115 & / & / \\
\hline $\begin{array}{l}\text { Goyal P, et al.(New } \\
\text { York City) }\end{array}$ & $\begin{array}{l}\text { Mar } 5 \text { to } \\
\text { Mar 27, } \\
2020\end{array}$ & $\begin{array}{l}\text { 62.2(48.6- } \\
\text { 73.7)_median(IQR) }\end{array}$ & 393 & 238 & / & / & / & / & 130_M92I \\
\hline $\begin{array}{l}\text { Gou FX, et al. } \\
\text { (Gansu Province) }\end{array}$ & $\begin{array}{l}\text { By Feb } \\
25,2020\end{array}$ & / & 91 & 39 & / & / & 24 & 44 & 23 \\
\hline $\begin{array}{l}\text { Fan Z, et al. } \\
\text { (Shanghai) }\end{array}$ & $\begin{array}{l}\text { Jan } 20 \text { to } \\
\text { Jan } 31 \\
2020\end{array}$ & 50 (36-64)_mean(IQR) & 148 & 75 & / & / & / & / & / \\
\hline Du Y, et al.(Wuhan) & $\begin{array}{l}\text { Jan } 9 \text { to } \\
\text { Feb 15, } \\
2020\end{array}$ & 65.8(14.2)_mean(SD) & 85 & 62 & / & / & / & / & / \\
\hline $\begin{array}{l}\text { Dong XC, et al. } \\
\text { (Tianjin) }\end{array}$ & / & 48.62(16.83)_mean(SD) & 135 & 72 & / & / & / & / & / \\
\hline $\begin{array}{l}\text { Dai H, et al. } \\
\text { (Jiangsu Province) }\end{array}$ & $\begin{array}{l}\text { Jan } 10 \text { to } \\
\text { Feb 7, } \\
2020\end{array}$ & / & 234 & 136 & / & / & 9 & 210 & 13 \\
\hline $\begin{array}{l}\text { Chu J, et al. } \\
\text { (Wuhan) }\end{array}$ & $\begin{array}{l}\text { Jan } 7 \text { to } \\
\text { Feb 11, } \\
2020\end{array}$ & $\begin{array}{l}\text { 39(26- } \\
\text { 73)_median(range) }\end{array}$ & 54 & 36 & / & / & / & 11_M6F5 & 43_M30_F \\
\hline $\begin{array}{l}\text { Chen T, et al. } \\
\text { (Wuhan) }\end{array}$ & $\begin{array}{l}\text { Jan } 1 \text { to } \\
\text { Feb 10, } \\
2020\end{array}$ & $\begin{array}{l}\text { 54(41- } \\
\text { 68)_median(IQR) }\end{array}$ & 203 & 108 & / & / & / & 96 & 73 \\
\hline $\begin{array}{l}\text { Cao J, et al. } \\
\text { (Wuhan) }\end{array}$ & $\begin{array}{l}\text { Jan } 3 \text { to } \\
\text { Feb } \\
1,2020\end{array}$ & $\begin{array}{l}\text { 54(37- } \\
67) \text { median(IQR) }\end{array}$ & 102 & 53 & / & / & l & / & / \\
\hline $\begin{array}{l}\text { Cai Q, et al. } \\
\text { (Shenzhen) }\end{array}$ & $\begin{array}{l}\text { Jan } 11 \text { to } \\
\text { Feb 6, } \\
2020\end{array}$ & $\begin{array}{l}\text { 47.5(33- } \\
\text { 61)_median(IQR) }\end{array}$ & 298 & 145 & / & / & / & 240_M106_F134 & 58_M39_F \\
\hline $\begin{array}{l}\text { Arentz M, et al. } \\
\text { (Washington State) }\end{array}$ & $\begin{array}{l}\text { Feb } 20 \text { to } \\
\text { Mar } 5 \text {, } \\
2020\end{array}$ & $\begin{array}{l}\text { 70(43- } \\
\text { 92)_median(IQR) }\end{array}$ & 21 & 11 & / & / & / & / & / \\
\hline $\begin{array}{l}\text { China CDC } \\
\text { (Mainland China) }\end{array}$ & $\begin{array}{l}\text { By Feb } \\
11,2020\end{array}$ & / & 44672 & 22981 & / & / & 36160 & & 6168 \\
\hline Fu S, et al.(Wuhan ) & $\begin{array}{l}\text { Feb } \\
9,2020\end{array}$ & $\begin{array}{l}64 \text { (37- } \\
\text { 87)_Mean(range) }\end{array}$ & 50 & 27 & / & / & / & / & 50_M27_F \\
\hline Fei J, et al.(Wuhan ) & $\begin{array}{l}\text { Jan } 1 \text { to } \\
\text { Jan } \\
30,2020\end{array}$ & $22-87$ & 192 & 97 & / & / & / & $85(44.3 \%)$ & $107\left(55.7^{\circ}\right.$ \\
\hline $\begin{array}{l}\text { Fu L, et al.(Wuhan, } \\
\text { Anhui) }\end{array}$ & / & / & 355 & 190 & / & / & / & 211 & 88 \\
\hline $\begin{array}{l}\text { Mazumder A, et al. } \\
\text { (India) }\end{array}$ & $\begin{array}{l}\text { as of Mar } \\
28,2020\end{array}$ & $\begin{array}{l}\text { 36(25- } \\
\text { 54)_median(IQR) }\end{array}$ & 413 & 274 & / & / & l & / & / \\
\hline $\begin{array}{l}\text { Tan L, et al. } \\
\text { (Wuhan) }\end{array}$ & $\begin{array}{l}\text { Jan } 14 \text { to } \\
\text { Mar } \\
14,2020\end{array}$ & / & 132 & 59 & / & / & / & 96_M36_F60 & 21_M11_F \\
\hline $\begin{array}{l}\text { Xiao G, et al. } \\
\text { (Wuhan) }\end{array}$ & $\begin{array}{l}\text { To March } \\
8,2020\end{array}$ & $\begin{array}{l}\text { 62(51- } \\
\text { 70)_median(IQR) }\end{array}$ & 287 & 160 & / & / & / & 163 & 124 \\
\hline $\begin{array}{l}\text { Wang Z, et al. } \\
\text { (Sichuan) }\end{array}$ & $\begin{array}{l}\text { Jan } 16 \text { to } \\
\text { Mar } \\
4,2020\end{array}$ & / & 538 & 285 & / & / & 450 & l & 88 \\
\hline $\begin{array}{l}\text { Petrilli CM, et al. } \\
\text { (New York City) }\end{array}$ & $\begin{array}{l}\text { March } 1 \\
\text { to Apr } \\
2,2020\end{array}$ & / & 4103 & 2072 & 89 & & l & 932_M560_F372 & / \\
\hline $\begin{array}{l}\text { Chen X, et al. } \\
\text { (Guangzhou) }\end{array}$ & $\begin{array}{l}\text { Jan } 20 \text { to } \\
\text { Mar 15, } \\
2020\end{array}$ & $\begin{array}{l}\text { 48(33- } \\
62) \_m e d i a n(I Q R)\end{array}$ & 284 & 131 & / & / & 22 & 199 & 40 \\
\hline $\begin{array}{l}\text { Jiang } X \text {, et al. } \\
\text { (Wuxi) }\end{array}$ & $\begin{array}{l}\text { Jan } 23 \text { to } \\
\text { Feb } \\
16,2020\end{array}$ & $\begin{array}{l}\text { 45(27- } \\
60) \text { median(range) }\end{array}$ & 55 & 27 & / & / & / & / & 47_M22_F \\
\hline
\end{tabular}




\begin{tabular}{|c|c|c|c|c|c|c|c|c|c|}
\hline Study & $\begin{array}{l}\text { collection } \\
\text { period }\end{array}$ & Age & $\begin{array}{l}\text { Case } \\
\text { load }\end{array}$ & $\begin{array}{l}\text { Male } \\
\text { (n) }\end{array}$ & $\begin{array}{l}\text { ICU- } \\
\text { Male }\end{array}$ & $\begin{array}{l}\text { ICU- } \\
\text { Female }\end{array}$ & mild & common & severe \\
\hline $\begin{array}{l}\text { Guo A-X, et al.(24 } \\
\text { Provinces) }\end{array}$ & $\begin{array}{l}\text { Dec 28, } \\
2019 \text { to } \\
\text { Mar } \\
4,2020\end{array}$ & / & 159 & 99 & / & / & l & / & l \\
\hline $\begin{array}{l}\text { Zheng Y, et al. } \\
\text { (Hangzhou) }\end{array}$ & $\begin{array}{l}\text { Jan } 22 \text { to } \\
\text { Mar } \\
5,2020\end{array}$ & $\begin{array}{l}66(58- \\
76) \_m e d i a n(I Q R)\end{array}$ & 34 & 23 & 23 & 11 & / & / & / \\
\hline $\begin{array}{l}\text { Shao Z, et al. } \\
\text { (Wuhan,Guangzhou } \\
\text { and Shenzhen) }\end{array}$ & $\begin{array}{l}\text { Dec,2019 } \\
\text { to } \\
\text { Mar,2020 }\end{array}$ & 58(46-69)_mean(IQR) & 325 & 189 & / & / & / & / & 222 \\
\hline $\begin{array}{l}\text { Lewnard JA, et al. } \\
(/)\end{array}$ & $\begin{array}{l}\text { By April } \\
9,2020\end{array}$ & $\begin{array}{l}\text { 60(47- } \\
\text { 72)_median(IQR) }\end{array}$ & 1277 & 725 & 158 & & / & / & / \\
\hline $\begin{array}{l}\text { Zeng } X \text {, et al.(Xi'an, } \\
\text { Beijing and Wuhan) }\end{array}$ & $\begin{array}{l}\text { Feb } 5 \text { and } \\
\text { Mar 20, } \\
2020\end{array}$ & / & 234 & 133 & I & / & 137_M74_F63 & / & 97_M59_F \\
\hline $\begin{array}{l}\text { Cummings MJ, et } \\
\text { al.(New York City) }\end{array}$ & $\begin{array}{l}\text { Mar. } 2 \text { to } \\
\text { April } \\
1,2020\end{array}$ & $\begin{array}{l}62(51- \\
72) \_m e d i a n(I Q R)\end{array}$ & 257 & 170 & / & / & / & / & / \\
\hline $\begin{array}{l}\text { Argenziano MG, et } \\
\text { al.(New York City) }\end{array}$ & $\begin{array}{l}\text { Mar. } 1 \text { to } \\
\text { April } \\
15,2020\end{array}$ & $\begin{array}{l}63(50- \\
75) \_ \text {median(IQR) }\end{array}$ & 1000 & 596 & 156 & 75 & / & / & l \\
\hline $\begin{array}{l}\text { Shi P, et al.(Shaanxi } \\
\text { Province) }\end{array}$ & $\begin{array}{l}\text { Jan } 23 \text { to } \\
\text { Mar } \\
7,2020\end{array}$ & $\begin{array}{l}46 \text { (34- } \\
58) \text { median(IQR) }\end{array}$ & 134 & 65 & / & / & l & 88_M40_F48 & 46_M25_F \\
\hline $\begin{array}{l}\text { Kong X, et al. } \\
\text { (Wuhan) }\end{array}$ & $\begin{array}{l}\text { Feb } 23 \text { to } \\
\text { Mar 13, } \\
2020\end{array}$ & $\begin{array}{l}49.98 \\
(13.73) \_m e a n(S D)\end{array}$ & 144 & 70 & / & / & / & / & / \\
\hline Huang $\mathrm{H}$, et al.(/) & $\begin{array}{l}\text { Jan } 20 \text { to } \\
\text { Feb } \\
29,2020\end{array}$ & / & 125 & 63 & / & / & 93_M47_F46 & / & 32_M16_F \\
\hline $\begin{array}{l}\text { Zhou F, et al. } \\
\text { (Yichang,Hubei) }\end{array}$ & $\begin{array}{l}\text { Jan } 17 \text { to } \\
\text { Feb 26, } \\
2020\end{array}$ & 55.94(18.83)_Mean(SD) & 197 & 98 & 56 & & / & / & / \\
\hline Xie J, et al.(Wuhan & / & $\begin{array}{l}\text { 62.0(50.8- } \\
\text { 71.0)_median(IQR) }\end{array}$ & 444 & 239 & I & I & I & / & / \\
\hline Zeng L, et al.(/) & $\begin{array}{l}\text { Jan } 11 \text { to } \\
\text { Feb } \\
29,2020\end{array}$ & 49 (14.5)_mean(SD) & 338 & 162 & / & I & / & 262_M115_F147 & 76_M47_F \\
\hline $\begin{array}{l}\text { Chen X, et al. } \\
\text { (Wuhan) }\end{array}$ & $\begin{array}{l}\text { Jan } 5 \text { to } \\
\text { Mar } 7 \\
2020\end{array}$ & $\begin{array}{l}\text { 51(35- } \\
\text { 66)_median(IQR) }\end{array}$ & 123 & 50 & / & / & / & / & 33 \\
\hline Li K, et al.(/) & $\begin{array}{l}\text { Jan } 31 \text { to } \\
\text { Mar 5, } \\
2020\end{array}$ & $\begin{array}{l}\text { 57(45- } \\
\text { 70)_median(range) }\end{array}$ & 102 & 59 & / & / & / & / & / \\
\hline $\begin{array}{l}\text { Yan D, et al. } \\
\text { (Wuhan) }\end{array}$ & $\begin{array}{l}\text { Jan } 31 \text { to } \\
\text { Mar } \\
9,2020\end{array}$ & $52(35-63)_{-}$ & 120 & 54 & / & / & / & 89 & 30 \\
\hline Wang Z, et al.(/) & $\begin{array}{l}\text { Feb } 23 \text { to } \\
\text { Mar 11, } \\
2020\end{array}$ & $\begin{array}{l}\text { 61.1(51.0- } \\
\text { 69.0)_median(IQR) }\end{array}$ & 116 & 65 & / & / & / & 101_M53_F48 & l \\
\hline $\begin{array}{l}\text { Liao X, et al. } \\
\text { (Sichuan) }\end{array}$ & / & $\begin{array}{l}50 \text { (39- } \\
65) \_m e d i a n(I Q R)\end{array}$ & 81 & 51 & 51 & & / & / & 81_M51_F \\
\hline $\begin{array}{l}\text { Zhang Y, et al. } \\
\text { (Wuhan) }\end{array}$ & $\begin{array}{l}\text { Jan } 29 \text { to } \\
\text { Feb 12, } \\
2020\end{array}$ & $\begin{array}{l}\text { 64(56- } \\
\text { 70)_median(IQR) }\end{array}$ & 258 & 138 & / & / & / & 87 & 116 \\
\hline Nie S, et al.(Wuhan) & $\begin{array}{l}\text { Feb } 9 \text { to } \\
\text { Feb 28, } \\
2020\end{array}$ & $\begin{array}{l}\text { 39(30- } \\
60) \text { _median(IQR) }\end{array}$ & 97 & 34 & / & / & 72_M21_F51 & / & 25_M13_F \\
\hline $\begin{array}{l}\text { Xu S, et al.(Wuhan, } \\
\text { Fuyang) }\end{array}$ & $\begin{array}{l}\text { Jan } 1 \text { to } \\
\text { Jan } \\
30,2020\end{array}$ & / & 355 & 193 & / & / & / & 224 & 60 \\
\hline Hu L, et al.(Wuhan) & $\begin{array}{l}\text { Jan } 8 \text { to } \\
\text { Mar } \\
10,2020\end{array}$ & $\begin{array}{l}61(23- \\
91) \_m e d i a n(\text { range) }\end{array}$ & 323 & 166 & / & I & I & 151_M75_F76 & 146_M77. \\
\hline
\end{tabular}




\begin{tabular}{|c|c|c|c|c|c|c|c|c|c|}
\hline Study & $\begin{array}{l}\text { collection } \\
\text { period }\end{array}$ & Age & $\begin{array}{l}\text { Case } \\
\text { load }\end{array}$ & $\begin{array}{l}\text { Male } \\
(n)\end{array}$ & $\begin{array}{l}\text { ICU- } \\
\text { Male }\end{array}$ & $\begin{array}{l}\text { ICU- } \\
\text { Female }\end{array}$ & mild & common & severe \\
\hline $\begin{array}{l}\text { Wen Y, et al. } \\
\text { (Shenzhen) }\end{array}$ & $\begin{array}{l}\text { Jan } 1 \text { to } \\
\text { Feb 28, } \\
2020\end{array}$ & 45.4(17.7)_mean(SD) & 417 & 197 & / & / & 381_M174_F207 & & 36_M23_F \\
\hline $\begin{array}{l}\text { Yan S, et al. } \\
\text { (Hainan) }\end{array}$ & $\begin{array}{l}\text { Jan } 22 \text { to } \\
\text { Mar } \\
14,2020\end{array}$ & $\begin{array}{l}\text { 51(36- } \\
\text { 62)_median(IQR) }\end{array}$ & 168 & 81 & / & / & / & 132_M60_F72 & 36_M21_F \\
\hline $\begin{array}{l}\text { Yang Y, et al. } \\
\text { (Wuhan) }\end{array}$ & $\begin{array}{l}\text { Feb } 12 \text { to } \\
\text { March } \\
2,2020\end{array}$ & 69.4(10.8)_Mean(SD) & 36 & 30 & / & / & / & l & / \\
\hline $\begin{array}{l}\text { Choe YJ, et al. } \\
\text { (Korea) }\end{array}$ & $\begin{array}{l}\text { as of Mar } \\
13 \text { th,2020 }\end{array}$ & / & 7755 & 2947 & I & / & / & l & / \\
\hline $\begin{array}{l}\text { Zhao W, et al. } \\
\text { (Beijing) }\end{array}$ & $\begin{array}{l}\text { Jan } 21 \text { to } \\
\text { Feb } \\
8,2020\end{array}$ & 52(20)_mean(SD) & 77 & 34 & / & / & / & 57_M23_F34 & 20_M11_F \\
\hline Fu L, et al.(Wuhan) & $\begin{array}{l}\text { Jan } 1 \text { to } \\
\text { Jan } \\
30,2020\end{array}$ & / & 200 & 99 & / & / & / & 88 & 58 \\
\hline $\begin{array}{l}\text { Chen L, et al. } \\
\text { (Wuhan) }\end{array}$ & $\begin{array}{l}\text { Feb } 1 \text { to } \\
\text { Mar 1, } \\
2020\end{array}$ & / & 534 & 268 & / & / & l & / & / \\
\hline $\begin{array}{l}\text { Liu Y, et al. } \\
\text { (Guangzhou) }\end{array}$ & $\begin{array}{l}\text { Jan } 10 \text { to } \\
\text { Feb } \\
24,2020\end{array}$ & $\begin{array}{l}\text { 48.1(34- } \\
62) \text { median(IQR) }\end{array}$ & 291 & 133 & 26 & & / & / & / \\
\hline Liu J, et al.(Wuhan) & $\begin{array}{l}\text { Jan } 16 \text { to } \\
\text { Feb } 15 \text {, } \\
2020\end{array}$ & $\begin{array}{l}\text { 35(29- } \\
\text { 43)_Median(IQR) }\end{array}$ & 64 & 23 & / & / & / & 63 & 1 \\
\hline Chen J, et al.(/) & / & 65.46(9.74)_Mean(SD) & 101 & 64 & 64 & 37 & / & / & / \\
\hline Wu Y, et al.(Wuhan) & $\begin{array}{l}\text { Dec } 2019 \\
\text { to Feb2, } \\
2020\end{array}$ & / & 297 & 147 & / & / & / & / & / \\
\hline $\begin{array}{l}\text { Xu Y, et al.(Wuhan, } \\
\text { Beijing,Shanghai) }\end{array}$ & $\begin{array}{l}\text { Feb } 7 \text { to } \\
\text { Feb } \\
28,2020\end{array}$ & $\begin{array}{l}57(43- \\
69) \text { median(IQR) }\end{array}$ & 69 & 35 & / & / & 44_M22_F22 & l & 25_M13_F \\
\hline Shi Q, et al.(Wuhan) & $\begin{array}{l}\text { By Feb } \\
15,2020\end{array}$ & $\begin{array}{l}\text { 71.0(59.0- } \\
\text { 80.0)_median (IQR) }\end{array}$ & 101 & 60 & / & / & / & / & / \\
\hline $\begin{array}{l}\text { Xu Y, et al. } \\
\text { (Guangzhou) }\end{array}$ & $\begin{array}{l}\text { Jan } 14 \text { to } \\
\text { Feb 20, } \\
2020\end{array}$ & 56.7 (15.4)_Mean(SD) & 45 & 29 & 29 & 16 & I & / & l \\
\hline $\begin{array}{l}\text { Zhang G, et al. } \\
\text { (Wuhan) }\end{array}$ & $\begin{array}{l}\text { Jan } 2 \text { to } \\
\text { Feb 10, } \\
2020\end{array}$ & $\begin{array}{l}\text { 55.0(39.0- } \\
\text { 66.5)_Median(IQR) }\end{array}$ & 221 & 108 & 22 & 10 & l & 166_M73_F93 & 55_M35_F \\
\hline $\begin{array}{l}\text { Cao M, et al. } \\
\text { (Shanghai) }\end{array}$ & $\begin{array}{l}\text { Jan } 20 \text { to } \\
\text { Feb } 15, \\
2020\end{array}$ & 50.1(16.3)_Mean(SD) & 198 & 101 & 17 & 2 & 3 & 176 & 9 \\
\hline $\begin{array}{l}\text { Chen X, et al. } \\
\text { (Hunan Province) }\end{array}$ & $\begin{array}{l}\text { Jan } 23 \text { to } \\
\text { Feb 14, } \\
2020\end{array}$ & $\begin{array}{l}\text { 46.0(34.0- } \\
\text { 59.0)_median(IQR) }\end{array}$ & 291 & 145 & / & / & 29_M16_F13 & 212_102F_110M & 50_M27_F \\
\hline $\begin{array}{l}\text { Wang } Y_{1} \text { et al. } \\
\text { (Wuhan) }\end{array}$ & $\begin{array}{l}\text { Jan } 1 \text { to } \\
\text { Feb 10, } \\
2020\end{array}$ & / & 110 & 48 & 48 & 62 & 72_M24_F48 & & 38_M24_F \\
\hline $\begin{array}{l}\text { Qi D, et al. } \\
\text { (Chongqing) }\end{array}$ & $\begin{array}{l}\text { Jan } 19 \text { to } \\
\text { Feb } 16, \\
2020\end{array}$ & $\begin{array}{l}\text { 48(20- } \\
\text { 80)_median(range) }\end{array}$ & 267 & 149 & / & / & 217_M110_F107 & & 50_M39_F \\
\hline $\begin{array}{l}\text { Huang Y, et al. } \\
\text { (Wuhan ) }\end{array}$ & $\begin{array}{l}\text { Jan } 21 \text { to } \\
\text { Feb } \\
10,2020\end{array}$ & 69.22(9.64)_mean(SD) & 36 & 25 & / & / & / & / & / \\
\hline $\begin{array}{l}\text { Xu L, et al. } \\
\text { (Wenzhou) }\end{array}$ & $\begin{array}{l}\text { As of Feb } \\
10,2020\end{array}$ & $\begin{array}{l}\text { 47(37- } \\
\text { 56)_Median(IQR) }\end{array}$ & 434 & 228 & / & l & / & / & 31 \\
\hline Zhang B, et al.(/) & $\begin{array}{l}\text { Jan } 11 \text { to } \\
\text { Feb 10, } \\
2020\end{array}$ & $\begin{array}{l}\text { 72.5(65.0- } \\
\text { 80.0)_median(IQR) }\end{array}$ & 82 & 54 & 14 & & / & / & I \\
\hline
\end{tabular}




\begin{tabular}{|c|c|c|c|c|c|c|c|c|c|}
\hline Study & $\begin{array}{l}\text { collection } \\
\text { period }\end{array}$ & Age & $\begin{array}{l}\text { Case } \\
\text { load }\end{array}$ & $\begin{array}{l}\text { Male } \\
(n)\end{array}$ & $\begin{array}{l}\text { ICU- } \\
\text { Male }\end{array}$ & $\begin{array}{l}\text { ICU- } \\
\text { Female }\end{array}$ & mild & common & severe \\
\hline Li J, et al.(/) & $\begin{array}{l}\text { From Feb } \\
8 \text { to Feb } \\
11,2020\end{array}$ & $\begin{array}{l}\text { 62.0(51.0- } \\
\text { 70.0)_median(IQR) }\end{array}$ & 47 & 28 & / & / & / & / & 47_M28_F \\
\hline $\begin{array}{l}\text { Liang Y, et al. } \\
\text { (Beijing) }\end{array}$ & $\begin{array}{l}\text { Jan21 to } \\
\text { Feb } \\
15,2020\end{array}$ & $\begin{array}{l}42.0(34.5- \\
66.0) \text { _median(IQR) }\end{array}$ & 21 & 11 & / & / & 18 & & 3 \\
\hline $\begin{array}{l}\text { Fan Z, et al. } \\
\text { (Shanghai) }\end{array}$ & $\begin{array}{l}\text { Jan } 20 \text { to } \\
\text { Jan } \\
31,2020\end{array}$ & $\begin{array}{l}50.5(36- \\
64) \text { median(IQR) }\end{array}$ & 148 & 73 & / & / & / & / & / \\
\hline Cao W(Xiangyang) & $\begin{array}{l}\text { Jan to } \\
\text { Feb } \\
16,2020\end{array}$ & / & 128 & 60 & / & / & 107_M48_F59 & & 21_M12_F \\
\hline $\begin{array}{l}\text { Jin J-M, et al. } \\
\text { (Wuhan) }\end{array}$ & $\begin{array}{l}\text { Jan } 29 \text { to } \\
\text { Feb 15, } \\
2020\end{array}$ & $\begin{array}{l}\text { 62(51- } \\
\text { 70)_median(range) }\end{array}$ & 43 & 22 & / & / & 13 & / & 14 \\
\hline Li X, et al.(Wuhan) & $\begin{array}{l}\text { Jan } 14 \text { to } \\
\text { Feb 13, } \\
2020\end{array}$ & 71.48(12.42)_mean(SD) & 25 & 10 & / & I & / & / & / \\
\hline $\begin{array}{l}\text { Wang L, et al. } \\
\text { (Wuhan) }\end{array}$ & $\begin{array}{l}\text { Jan } 14 \text { to } \\
\text { Feb } \\
13,2020\end{array}$ & $\begin{array}{l}\text { 54(38- } \\
69) \text { median(IQR) }\end{array}$ & 116 & 67 & 6 & 5 & 59_M34_F25 & / & 46_M27_F \\
\hline $\begin{array}{l}\text { Ai J, et al. } \\
\text { (Xiangyang) }\end{array}$ & $\begin{array}{l}\text { Until Feb } \\
10,2020\end{array}$ & 50.38(16.86)_mean(SD) & 102 & 52 & 8 & & / & / & / \\
\hline $\begin{array}{l}\text { Lei Liu, et al. } \\
\text { (Chongqing) }\end{array}$ & $\begin{array}{l}\text { Jan } 20 \text { to } \\
\text { Feb 3, } \\
2020\end{array}$ & $\begin{array}{l}\text { 45(34- } \\
\text { 51)_median(IQR) }\end{array}$ & 51 & 32 & / & / & 44_M28_F16 & & 7_M4_F3 \\
\hline Liu Y, et al.(/) & $\begin{array}{l}\text { Jan } 2 \text { to } \\
\text { Feb 1, } \\
2020\end{array}$ & $\begin{array}{l}\text { 55(43- } \\
66) \text { median(IQR) }\end{array}$ & 109 & 59 & / & / & / & 56_M31_F25 & 53_M28_F \\
\hline Wu C, et al.(Wuhan) & $\begin{array}{l}\text { Dec 25, } \\
2019 \text { to } \\
\text { Jan 26, } \\
2020\end{array}$ & $\begin{array}{l}51(43- \\
60) \_ \text {Median(IQR) }\end{array}$ & 201 & 128 & $53(26.4 \%)$ & & $44(21.9 \%)$ & / & 84 _M60 _ \\
\hline Shi H, et al.(Wuhan) & $\begin{array}{l}\text { Dec 20, } \\
2019 \text { to } \\
\text { Jan } \\
23,2020\end{array}$ & 49.5(11.0)__Mean(SD) & 81 & 42 & / & / & / & / & / \\
\hline $\begin{array}{l}\text { Pan Y, et al. } \\
\text { (Wuhan) }\end{array}$ & $\begin{array}{l}\text { Dec } \\
30,2019 \\
\text { to Jan } \\
31,2020\end{array}$ & 44.9(15.2)_Mean(SD) & 63 & 33 & / & l & I & / & / \\
\hline $\begin{array}{l}\text { Wang K, et al. } \\
\text { (Xiaogan) }\end{array}$ & $\begin{array}{l}\text { Jan } \\
25,2020 \\
\text { to Feb } \\
9,2020\end{array}$ & $\begin{array}{l}\text { 53(32- } \\
\text { 78)_Median(Range) }\end{array}$ & 114 & 58 & / & / & / & / & / \\
\hline $\begin{array}{l}\text { Shi Y, et al. } \\
\text { (Zhejiang Province) }\end{array}$ & $\begin{array}{l}\text { To Feb } \\
17,2020\end{array}$ & / & 487 & 259 & / & / & 438_M223_F215 & / & 49_M36_F \\
\hline $\begin{array}{l}\text { McMichael TM, et } \\
\text { al.(Washington) }\end{array}$ & $\begin{array}{l}\text { Feb } 28 \text { to } \\
\text { Mar } \\
18,2020\end{array}$ & $\begin{array}{l}\text { 72(21- } \\
\text { 100)_Median(Range) }\end{array}$ & 167 & 55 & / & l & / & / & / \\
\hline $\begin{array}{l}\text { Jin X, et al. } \\
\text { (Zhejiang Province) }\end{array}$ & $\begin{array}{l}\text { Jan } 17 \text { to } \\
\text { Feb 8, } \\
2020\end{array}$ & / & 651 & 331 & $17(2.6 \%)$ & & / & / & / \\
\hline $\begin{array}{l}\text { Chen N, et al. } \\
\text { (Wuhan) }\end{array}$ & $\begin{array}{l}\text { Jan } 1 \text { to } \\
\text { Jan 20, } \\
2020\end{array}$ & 55.5 (13.1)_Mean (SD) & 99 & 67 & / & / & / & / & / \\
\hline $\begin{array}{l}\text { Zhou F, et al. } \\
\text { (Wuhan) }\end{array}$ & $\begin{array}{l}\text { By Jan } \\
31,2020\end{array}$ & $\begin{array}{l}56.0 \text { (46.0- } \\
67.0) \_ \text {Median(IQR) }\end{array}$ & 191 & 119 & / & l & I & / & / \\
\hline $\begin{array}{l}\text { Zhang J-J, et al. } \\
\text { (Wuhan) }\end{array}$ & $\begin{array}{l}\text { Jan } 16 \text { to } \\
\text { Feb } \\
3,2020\end{array}$ & $\begin{array}{l}57 \text { (25- } \\
\text { 7)_Median(range) }\end{array}$ & 140 & 71 & / & / & $82(58.6 \%)$ & & $58(41.4 \%$ \\
\hline $\begin{array}{l}\text { Zhang G, et al. } \\
\text { (Wuhan) }\end{array}$ & $\begin{array}{l}\text { Jan } 16 \text { to } \\
\text { Feb } \\
25,2020\end{array}$ & $\begin{array}{l}49.0 \text { (39.0- } \\
58.0 \text { )_Median(Range) }\end{array}$ & 95 & 53 & / & / & 63_M32_F31 & & 32_M21_F \\
\hline
\end{tabular}




\begin{tabular}{|c|c|c|c|c|c|c|c|c|c|}
\hline Study & $\begin{array}{l}\text { collection } \\
\text { period }\end{array}$ & Age & $\begin{array}{l}\text { Case } \\
\text { load }\end{array}$ & $\begin{array}{l}\text { Male } \\
(n)\end{array}$ & $\begin{array}{l}\text { ICU- } \\
\text { Male }\end{array}$ & $\begin{array}{l}\text { ICU- } \\
\text { Female }\end{array}$ & mild & common & severe \\
\hline $\begin{array}{l}\text { Yang W, et al. } \\
\text { (Wenzhou) }\end{array}$ & $\begin{array}{l}\text { Jan } 17 \text { to } \\
\text { Feb 10, } \\
2020\end{array}$ & $\begin{array}{l}45.11 \\
(13.35) \_M e a n(S D)\end{array}$ & 149 & 81 & 0 & 0 & / & / & / \\
\hline $\begin{array}{l}\text { Xu X-W, et al. } \\
\text { (Zhejiang Province) }\end{array}$ & $\begin{array}{l}\text { Jan } 10 \text { to } \\
\text { Jan } 26 \text {, } \\
2020\end{array}$ & 41(32-52) Median(IQR) & 62 & 35 & / & / & / & / & / \\
\hline $\begin{array}{l}\text { Wu J, et al.(Jiangsu } \\
\text { Province) }\end{array}$ & $\begin{array}{l}\text { Jan } 22 \text { to } \\
\text { Feb } \\
14,2020\end{array}$ & $\begin{array}{l}\text { 46.1 } \\
(15.42) \_M e d i a n(S D)\end{array}$ & 80 & 39 & / & / & $28(35 \%)$ & $49(61.25 \%)$ & $3(3.75 \%)$ \\
\hline $\begin{array}{l}\text { Wang Z, et al. } \\
\text { (Wuhan) }\end{array}$ & $\begin{array}{l}\text { Jan } 16 \text { to } \\
\text { Jan } 29 \text {, } \\
2020\end{array}$ & $\begin{array}{l}\text { 42.0 (35.0- } \\
62.0) \_ \text {Median(IQR) }\end{array}$ & 69 & 32 & / & / & / & / & / \\
\hline $\begin{array}{l}\text { Wang } D \text {, et al. } \\
\text { (Wuhan) }\end{array}$ & $\begin{array}{l}\text { Jan } 1 \text { to } \\
\text { Jan 28, } \\
2020\end{array}$ & $\begin{array}{l}56 \text { (42-68)_Median } \\
\text { (IQR) }\end{array}$ & 138 & 75 & $\begin{array}{l}22 \\
(61.1 \%)\end{array}$ & $\begin{array}{l}14 \\
(38.9 \%)\end{array}$ & / & / & I \\
\hline $\begin{array}{l}\text { Wan S, et al. } \\
\text { (Chongqing) }\end{array}$ & $\begin{array}{l}\text { Jan } 23 \text { to } \\
\text { Feb } 8 \\
2020\end{array}$ & $\begin{array}{l}47 \text { (36- } \\
\text { 55)_Median(IQR) }\end{array}$ & 135 & 72 & l & I & 95_M52_F43 & / & 40-M21_F \\
\hline $\begin{array}{l}\text { Tian S, et al. } \\
\text { (Beijing) }\end{array}$ & $\begin{array}{l}\text { By Feb } \\
10,2020\end{array}$ & $\begin{array}{l}47.5(1- \\
94) \_M e d i a n(\text { range })\end{array}$ & 262 & 127 & / & / & I & $216(82.4 \%)$ & $46(17.6 \%$ \\
\hline $\begin{array}{l}\text { Qian G-Q, et al. } \\
\text { (Zhejiang Province) }\end{array}$ & $\begin{array}{l}\text { Jan } 20 \text { to } \\
\text { Feb 11, } \\
2020\end{array}$ & $\begin{array}{l}50 \text { (36.5- } \\
\text { 57)_Median(IQR) }\end{array}$ & 91 & 37 & / & / & / & / & / \\
\hline $\begin{array}{l}\text { Liu K, et al.(Hubei } \\
\text { Province) }\end{array}$ & $\begin{array}{l}\text { Dec } 30 \\
2019 \text { to } \\
\text { Jan } 24 \\
2020\end{array}$ & $\begin{array}{l}\text { 57(20-83)_Median } \\
\text { (range) }\end{array}$ & 137 & 61 & / & / & / & / & / \\
\hline $\begin{array}{l}\text { Liu K, et al.(Hainan } \\
\text { Province) }\end{array}$ & $\begin{array}{l}\text { Jan } 15 \text { to } \\
\text { Feb } 18\end{array}$ & $\begin{array}{l}\text { Elderly_68(65.25- } \\
69.75) \text { YYoung and } \\
\text { middle-aged } 47(35.75- \\
51.25)\end{array}$ & 56 & 31 & / & / & / & / & / \\
\hline $\begin{array}{l}\text { Lian J, et al. } \\
\text { (Zhejiang Province) }\end{array}$ & $\begin{array}{l}\text { Jan } 17 \text { to } \\
\text { Feb } \\
12,2020\end{array}$ & / & 788 & 407 & $24(3.05 \%)$ & & 710(90.1\%) & / & $61(7.7 \%)$ \\
\hline Li Q, et al.(Wuhan) & $\begin{array}{l}\text { By Jan } \\
22,2020\end{array}$ & $\begin{array}{l}59 \text { (15-89)_Median } \\
\text { (range) }\end{array}$ & 425 & 240 & / & / & l & / & / \\
\hline $\begin{array}{l}\text { Lescure FX, et al. } \\
\text { (Paris) }\end{array}$ & $\begin{array}{l}\text { Jan } 24 \text { to } \\
\text { Jan } \\
29,2020\end{array}$ & 46.0 (20.2)_Median(SD) & 5 & 3 & $3(0.6)$ & $2(0.4)$ & / & / & / \\
\hline $\begin{array}{l}\text { Huang Y, et al. } \\
\text { (Wuhan) }\end{array}$ & $\begin{array}{l}\text { Dec } \\
21,2019 \\
\text { to Jan } \\
28,2020\end{array}$ & $\begin{array}{l}56.24 \\
(17.14) \_M e d i a n(S D)\end{array}$ & 34 & 14 & $8(24.2 \%)$ & & / & / & l \\
\hline $\begin{array}{l}\text { Huang } C \text {, et al. } \\
\text { (Wuhan) }\end{array}$ & $\begin{array}{l}\text { By Jan 2, } \\
2020\end{array}$ & $\begin{array}{l}49(41-58) \_ \text {Median } \\
\text { (IQR) }\end{array}$ & 41 & 30 & $\begin{array}{l}11 \\
(84.6 \%)\end{array}$ & 2 & / & / & / \\
\hline $\begin{array}{l}\text { Guan W-J, et al.(30 } \\
\text { Pro. in China) }\end{array}$ & $\begin{array}{l}\text { Dec } \\
11,2019 \\
\text { to Jan } 29 \\
2020\end{array}$ & $\begin{array}{l}47.0 \text { (35.0 } \\
58.0) \_ \text {Median (IQR) }\end{array}$ & 1099 & 640 & / & / & l & / & / \\
\hline $\begin{array}{l}\text { Easom N,et al. } \\
\text { (United Kingdom) }\end{array}$ & $\begin{array}{l}\text { Jan } 29 \text { to } \\
\text { Feb } \\
24,2020\end{array}$ & $0-76$ & 68 & 32 & / & / & / & / & / \\
\hline $\begin{array}{l}\text { Deng } Y \text {, et al. } \\
\text { (Wuhan) }\end{array}$ & $\begin{array}{l}\text { Jan } 1 \text { to } \\
\text { Feb } \\
21,2020\end{array}$ & / & 225 & 124 & / & / & / & / & 104 \\
\hline $\begin{array}{l}\text { Chen T, et al. } \\
\text { (Wuhan) }\end{array}$ & $\begin{array}{l}\text { until Feb } \\
28,2020\end{array}$ & $\begin{array}{l}62.0 \text { (44.0- } \\
\text { 70.0)_Median(IQR) }\end{array}$ & 274 & 171 & / & / & / & / & / \\
\hline $\begin{array}{l}\text { Chen } L \text {, et al. } \\
\text { (Wuhan) }\end{array}$ & $\begin{array}{l}\text { Jan } 14 \text { to } \\
29,2020\end{array}$ & $\begin{array}{l}56(26- \\
79) \_ \text {Median(Range) }\end{array}$ & 29 & 21 & / & / & / & 15 & 9 \\
\hline $\begin{array}{l}\text { Chen J, et al. } \\
\text { (Shanghai) }\end{array}$ & $\begin{array}{l}\text { Jan } 20 \text { to } \\
\text { Feb 6, } \\
2020\end{array}$ & $\begin{array}{l}\text { 51(36- } \\
\text { 64)_Median(IQR) }\end{array}$ & 249 & 126 & $22(8.8 \%)$ & & / & / & / \\
\hline
\end{tabular}




\begin{tabular}{|c|c|c|c|c|c|c|c|c|c|}
\hline Study & $\begin{array}{l}\text { collection } \\
\text { period }\end{array}$ & Age & $\begin{array}{l}\text { Case } \\
\text { load }\end{array}$ & $\begin{array}{l}\text { Male } \\
(n)\end{array}$ & $\begin{array}{l}\text { ICU- } \\
\text { Male }\end{array}$ & $\begin{array}{l}\text { ICU- } \\
\text { Female }\end{array}$ & mild & common & severe \\
\hline $\begin{array}{l}\text { Chang, et al. } \\
\text { (Beijing) }\end{array}$ & $\begin{array}{l}\operatorname{Jan} 16 \text { to } \\
\operatorname{Jan} \\
29,2020\end{array}$ & $\begin{array}{l}\text { 34(34- } \\
\text { 48)_Median(25th-75th } \\
\text { percentile) }\end{array}$ & 13 & 10 & I & I & I & I & I \\
\hline $\begin{array}{l}\text { Chen G, et al. } \\
\text { (Wuhan) }\end{array}$ & $\begin{array}{l}\text { By Jan } \\
27,2020\end{array}$ & $\begin{array}{l}\text { 56.0(50.0- } \\
\text { 65.0)_Median(IQR) }\end{array}$ & 21 & 17 & / & / & I & 10_M7_F3 & 11_M10_F \\
\hline $\begin{array}{l}\text { Liu W, et al. } \\
\text { (Wuhan) }\end{array}$ & $\begin{array}{l}\text { Dec 30, } \\
2019 \text { to } \\
\text { Jan } 15 \\
2020\end{array}$ & $\begin{array}{l}38 \text { (33- } \\
\text { 57)_Median(IQR) }\end{array}$ & 78 & 39 & I & / & I & 70 (89.7\%) & $8(10.3 \%)$ \\
\hline Feng $\mathrm{Y}$, et al.(China) & $\begin{array}{l}\text { Jan } 1 \text { to } \\
\text { Feb 15, } \\
2020\end{array}$ & $\begin{array}{l}53(40- \\
64) \_ \text {Median(IQR) }\end{array}$ & 476 & 271 & / & I & I & 352_M190_F162 & 54_M33_ \\
\hline $\begin{array}{l}\text { Grasselli G, et al. } \\
\text { (Italy) }\end{array}$ & $\begin{array}{l}\text { Feb } 20 \text { to } \\
\text { March } \\
18,2020\end{array}$ & $\begin{array}{l}\text { 63(56- } \\
70) \_ \text {Median(IQR) }\end{array}$ & 1591 & 1304 & 1304 & 287 & I & I & I \\
\hline
\end{tabular}

The main characteristics and demographic data of the included studies are listed in Table 1. As of April 27, 2020, 96237 patients were included in this metaanalysis, and $46516(48.3 \%)$ were women. The results of the random effects model meta-analysis showed that in the sex distribution of COVID-19 females accounted for 0.47 [95\% $\mathrm{Cl}(0.45-0.48)]$ (Fig. 2S-A), which was lower than males 0.53 [95\% $\mathrm{Cl}(0.52-0.55)$ ]. Funnel plot was used to test publication bias (Fig. 2S-B), indicating no notable evidence of publication bias. The sensitivity analysis based on the random effects model showed that there was not a particular article that greatly affected the result of this meta-analysis, suggesting a stable result (Table 2).

Table 2

Begg's and Egger's test results for all outcomes.

\begin{tabular}{|llll|}
\hline & Included studies & Begg's test $\boldsymbol{p}$ value & Egger's test $\boldsymbol{p}$ value \\
\hline Proportion of female patients & 156 & 0.2827 & 0.0346 \\
\hline Proportion of female patients admitted into ICU or with severe/critical illness & 67 & 0.2743 & 0.3686 \\
Fatality rate of female patients & 25 & 0.4688 & 0.9036 \\
\hline
\end{tabular}

Females admitted into ICU or with severe/critical illness were also analyzed, the ratio was 0.14 [95\% $\mathrm{Cl}(0.11-0.17)]$ among all the severe/critically ill patients, which was much lower than males 0.24 [95\% $\mathrm{Cl}(0.19-0.29)]$ (Fig. 2-A). The funnel plot (Fig. 2-B) also showed no significant publication bias. As to the fatality rate of the females, the meta-analysis of the female fatality rate was shown in Fig. 2-C and Fig. 2-D, indicating that the overall female fatality rate was 0.10 [95\% Cl (0.05-0.17)], with male fatality rate 0.18 [95\% $\mathrm{Cl}(0.11-0.29)]$. In conclusion, female patients are less likely to be infected with COVID-19 than are males, the ratio of females admitted into ICU or with serve/critical illness, and the female fatality rate are lower than those of males.

The results of the Begg's and Egger's tests are displayed in Table 2. There were publication biases in the meta-analysis of female prevalence $(p=0.0346)$ by using Egger's test. Figures 3-A, B, C present the relative publication bias plots according to Begg's tests as examples. No remarkable alterations of the merged proportion and corresponding $95 \% \mathrm{Cl}$ value were observed when each individual study was removed one by one in the sensitivity analysis (Fig. 3-D, E, F), confirming the aforementioned stability of the results.

\section{Discussion}

From the above meta-analysis of sex difference in COVID-19, 156 reported studies were selected from hospitals in China, United States, United Kingdom, France, Italy, Korea, Singapore and India, respectively. From this analysis, we observed that more men than women were diagnosed with COVID-19. Also, male patients suffered of more severe symptoms than did female patients, and their mortality rate was twice greater than that of female patients. Moreover, recent findings from Li et al. [9] indicated that male COVID-19 patients are more likely to have complicated clinical conditions and worse prognosis as compared to females, such as secondary infection and heart function damage (preprint). SARS-CoV-2 belongs to the genus Betacoronavirus and shared $79.5 \%$ of the sequence with SARS-CoV and about $50 \%$ with MERS-CoV [10]. Tracing back to the history of SARS in 2003, males (21.9\%) had a significantly higher fatality rate $(p<0.0001)$ than did females $(13.2 \%)$ in the 299 deaths among 1755 SARS cases. The relative risk was $1.62(95 \% \mathrm{Cl}: 1.21,2.16)$ after adjustment for age [11]. Other studies indicated that there also exist sex differences in the outbreak of MERS (Middle East respiratory syndrome) in Saudi Arabia and South Korea. Al-Raddadi et al. [12] studied that in Saudi Arabia from 2012 to 2015, males were more susceptible than females (63.2\% versus $36.8 \%$ ) of all the confirmed patients $(n=835)$. The fatality rate of men is 1.4 times great than that of women $(47.9 \%$ versus $34.9 \%)$, which, though, is not statistically significant. Among the 186 MERS cases in South Korea, 111 (59.7\%) were males and 75 (40.3\%) were females, and the fatality rate of men (23.4\%) is also greater than that of women (16\%) [13]. The sex differs in the intensity, prevalence, and pathogenesis of epidemic-prone infectious diseases caused by viruses, bacteria, parasites and fungi, with higher prevalence of infections for males than for females [14].

All of these disparities raise an interesting question: What causes the observed sex predisposition in the coronavirus infection? In the following discussion, we will discuss the possible causes of sex differences in the case of coronavirus infections. 
It was recently found that ACE2 serves as the main entry of SARS-CoV-2 into host cells while the transmembrane protease, serine 2 (TMPRSS2) is for S protein priming of this virus [5]. Both genes might serve as the good candidates to explain sex-related effects, as ACE2 is located on the X chromosome and TMPRSS2 was found to participate in the non-canonical activation pathway of ERß2 by estradiol [15]. Asselta et al (preprint) [16] reported that TMPRSS2 variants and genetic expression levels might play a role in COVID-19 severity by investigating GTEx and GEO databases. One distinct haplotype has been functionally linked to eQTL (rs8134378), which could be regulated through androgens, possibly explaining the sex bias in COVID-19 severity. However, no significant evidence was found for ACE2 to be associated with COVID-19 severity/sex bias among Italian patients. Lazzeri et al [17] indicated that a reduced androgen stimulation might indeed represent a protective factor for males from an observational study in male population in Lombardy (preprint). Host genetic background is considered to influence the severity of SARS and MERS [18]. Thus, based on currently limited studies, we could not come up with any reasonable genetic explanations for the sex difference in COVID-19 incidents, severity and fatality, and further experimental and clinical studies are needed to achieve this goal.

\section{Sex-specific immune-defense factors}

By detecting the difference of SARS-CoV-2 IgG antibody between male and female patients, the plasmas from 331 enrolled patients were analyzed by a study. Most severely ill female patients exhibited a high level of IgG antibody compared to severely ill male patients. In the early phase of COVID-19 progression, the production of IgG antibody in females tended to be stronger than that in male patients (preprint) [19]. However, the exact mechanism remains elusive. Males and females show differences in immunological responses, in which both genes and hormones are engaged. Generally, women have a stronger innate and adaptive (humoral and cellular) immune response than do men [20].

The sex-specific function of innate detection of nucleic acids is mainly accomplished through pattern recognition receptors (PRRs). By infecting Seoul virus in Norway rats, Hannah et al [21] found that expression of antiviral genes (MYD88, IRF7,RIG1, JAK2, STAT3, IFN 3 and Mx2) was higher in the lungs of females than that in males' lungs. Putative androgen response elements (AREs) and estrogen response elements (EREs) are involved in the promoters of several antiviral genes. As to the adaptive immune system, females generally exhibit higher levels of the adaptive immune response to antigenic stimulation, vaccination and infection than do males [22]. Compared to age-matched males, females had higher CD4/CD8 ratios and helper T cell type 1 (Th1) responses, while men had higher $\mathrm{CD}^{+} \mathrm{T}$ cell frequencies. Mouse studies also revealed that female mice have higher proportions of Th1, helper T cell type 2 (Th2) and regulatory $\mathrm{T}$ cell responses than do male mice. Cytotoxic $\mathrm{T}$ cell activity was in a higher level with an up-regulated expression pattern of antiviral and proinflammatory genes in females compared with males, many of which have estrogen-response elements in their promoters [23]. These studies suggest that females possess a much stronger immune system than do males, though more in-dept and systematic studies are needed to validate this statement pertinent to the infection of SARS-CoV-2.

\section{Sex hormone regulation}

The putative hormone receptors expressed in the cells of the immune system mainly include estrogen receptor alpha (ERa) and estrogen receptor beta (ER $\beta$ ), androgen receptor (AR) and progesterone receptor (PR) [24].

To date, it is widely documented that in murine models and humans, there are sex differences in the incidence and severity of respiratory viral infections [11] [12] [13] [25]. Sex hormone receptor activity is correlated with the sex differences in immune cell number and functional responses in the respiratory tract. For instance, Channappanavar et al [25] reported that male mice are more susceptible to SARS-CoV infection than are female mice of the same age group. Ovariectomy or adding estrogen receptor (ER) antagonist increased the mortality of female mice, suggesting that the ER signaling pathway may play a role in protecting females in coronavirus infection. ERs are expressed in various lymphoid tissue cells, in lymphocytes, macrophages, and dendritic cells (DCs). Through ovariectomized mice, the amount of inflammatory monocyte macrophages (IMMs) increased compared to intact female mice, suggesting that estrogen signaling in females inhibited the accumulation and function of IMMs in the lungs and might exert protection function in females [25].

\section{Behavioral factors}

The aforementioned sex predisposition might be associated with the higher smoking rate in men. In China 2015, smoking prevalence was $27.7 \%$ (52.1\% among men and $2.7 \%$ among women) [26], showing a significant sex difference. From the latest report of the European Centre for Disease Control and Prevention (ECDC), smokers are more susceptible to serious complications from a coronavirus infection (data unpublished). Smokers have also appeared to be more susceptible to severe acute respiratory syndrome. A latest systematic review declared that smoking is most likely associated with the negative progression and adverse outcomes of COVID-19, yet further research is needed [27]. Notably, Guo et al (preprint) [28] reported that there was significantly higher expression of 2019-nCoV receptor, ACE2 in smoker lung samples compared to non-smoker lung samples by using RNA-seq and DNA microarray datasets. However, significant disparity wasn't found between sex groups (male vs female), which needs to be further validated.

In Saudi Arabia, because of the complex culturally-specific social phenomenon for women, sex-segregation is strictly enacted in the country. Saudi Arabian women's roles are largely defined within familial spaces, with the concept of modesty by wearing unique cultural customs (purdah and veil). As a result, this may unintentionally serve to reduce the risk of women contracting the air-borne transmission of respiratory diseases like MERS [29].

\section{Strengths and Limitations}

The main strength of this study is that we have provided thus far the largest series analysis of the association between biological sex and severity in COVID19 patients. However, the main limitation is that more specific and high-quality literature on this topic is still urgently needed.

\section{Conclusions}


In conclusion, from our meta-analysis of a large cohort of clinical studies on COVID-19 patients from multiple countries, we can conclude that females are less severely affected by SARS-CoV-2, and sex difference may exist in severely/critically ill and deceased COVID-19 patients. The possible influence factors on this sex difference may include genetic, hormonal, immune and behavioral ones. However, as there lacks the sex-specific clinical data, partly due to the possibility of that sex has not regularly been considered a biological variable for analysis in clinic, additional large-scale clinical and basic studies are required to further investigate the impact of sex on the incidents of and treatments for COVID-19 and to better understand molecule mechanism underlying this sex difference. These lines of information will be vitally important for the health sectors in the world to develop better strategies or approaches for future prevention, diagnosis, and treatment of COVID-19 for both male and female patients.

\section{Abbreviations}

\section{ACE2}

Angiotensin-converting enzyme 2;

AHRQ

Agency for Healthcare Research and Quality;

AR

androgen receptor;

ARDS

acute respiratory distress syndrome;

AREs

androgen response elements;

DCs

dendritic cells;

ECDC

European Centre for Disease Control and Prevention;

ER

estrogen receptor;

EREs

estrogen response elements;

Era

estrogen receptor alpha;

$\mathrm{Er} \beta$

estrogen receptor beta;

ICU

Intensive care unit;

IMMs

inflammatory monocyte macrophages;

MERS

Middle East Respiratory Syndrome;

MODS

multiple organ dysfunction syndromes;

PR

progesterone receptor;

PRISMA

Preferred Reporting for Systematic Reviews of Meta-analyses;

PRRs

pattern recognition receptors;

WHO

World Health Organization

\section{Declarations}

\section{Ethics approval and consent to participate}

Not applicable

\section{Consent for publication}

Not applicable

\section{Availability of data and materials}

Not applicable 


\section{Competing interests}

Disclosure: The authors declare that there is no competing of interests.

Funding: This work was supported by grants from the Clinical Research Pilot Project of Tongji hospital, Huazhong University of Science and Technology (No. 2019CR205). The funders had no role in study design, data collection, analysis, interpretation of data and in writing the manuscript.

\section{Authors' contributions}

SW and JZ conceived and coordinated the study; LM and HL designed and did the literature review and extracted the data with help from HL; LX analyzed the data. LM, HL, MW, LX, QZ and JZ interpreted the data. LM wrote the initial draft of the manuscript with input from LX; All authors revised the subsequent drafts.

\section{Acknowledgements}

We would like to thank the funding agency of Tongji hospital of Huazhong University of Science and Technology for supporting this study.

\section{Authors' information}

All authors are in Department of Obstetrics and Gynecology, Tongji Hospital, Tongji Medical College, Huazhong University of Science and Technology, Wuhan, Hubei, China.

\section{References}

1. Huang C, Wang Y, Li X, Ren L, Zhao J, Hu Y, Zhang L, Fan G, Xu J, Gu X et al: Clinical features of patients infected with 2019 novel coronavirus in Wuhan, China. The Lancet 2020, 395(10223):497-506.

2. Lu R, Zhao X, Li J, Niu P, Yang B, Wu H, Wang W, Song H, Huang B, Zhu N et al: Genomic characterisation and epidemiology of 2019 novel coronavirus: implications for virus origins and receptor binding. Lancet 2020, 395(10224):565-574.

3. Guan W-J, Ni Z-Y, Hu Y, Liang W-H, Ou C-Q, He J-X, Liu L, Shan H, Lei C-L, Hui DSC et al: Clinical Characteristics of Coronavirus Disease 2019 in China. The New England journal of medicine 2020.

4. Wang Z, Yang B, Li Q, Wen L, Zhang R: Clinical Features of 69 Cases with Coronavirus Disease 2019 in Wuhan, China. Clinical infectious diseases : an official publication of the Infectious Diseases Society of America 2020.

5. Hoffmann M, Kleine-Weber H, Schroeder S, Krüger N, Herrler T, Erichsen S, Schiergens TS, Herrler G, Wu N-H, Nitsche A et al: SARS-CoV-2 Cell Entry Depends on ACE2 and TMPRSS2 and Is Blocked by a Clinically Proven Protease Inhibitor. Cell 2020.

6. Zhao Y, Zhao Z, Wang Y, Zhou Y, Ma Y, Zuo W: Single-cell RNA expression profiling of ACE2, the putative receptor of Wuhan 2019-nCov. 2020:2020.2001.2026.919985.

7. Moher D, Liberati A, Tetzlaff J, Altman DG: Preferred reporting items for systematic reviews and meta-analyses: the PRISMA statement. BMJ 2009, 339:b2535

8. Celiac Disease. Rockville (MD): Agency for Healthcare Research and Quality (US); 2004 Sep. (Evidence Reports/Technology Assessments, No. 104.) Appendix D. Quality Assessment Forms.

9. Li J, Zhang Y, Wang F, Liu B, Li H, Tang G, Chang Z, Liu A, Fu C, Gao J et al: Sex differences in clinical findings among patients with coronavirus disease 2019 (COVID-19) and severe condition Preprint at https://www.medrxiv.org/content/10.1101/2020.02.27.20027524v1.full.pdf. Accessed 29 February 2020

10. Wang H, Li X, Li T, Zhang S, Wang L, Wu X, Liu J: The genetic sequence, origin, and diagnosis of SARS-CoV-2. Eur J Clin Microbiol Infect Dis 2020:1-7.

11. Karlberg J, Chong DSY, Lai WYY: Do men have a higher case fatality rate of severe acute respiratory syndrome than women do? Am J Epidemio/ 2004 , 159(3):229-231.

12. Al-Raddadi RM, Shabouni OI, Alraddadi ZM, Alzalabani AH, Al-Asmari AM, Ibrahim A, Almarashi A, Madani TA: Burden of Middle East respiratory syndrome coronavirus infection in Saudi Arabia. J Infect Public Health 2019.

13. Kim KH, Tandi TE, Choi JW, Moon JM, Kim MS: Middle East respiratory syndrome coronavirus (MERS-CoV) outbreak in South Korea, 2015: epidemiology, characteristics and public health implications. J Hosp Infect 2017, 95(2):207-213.

14. vom Steeg LG, Klein SL: SeXX Matters in Infectious Disease Pathogenesis. PLoS Pathog 2016, 12(2):e1005374.

15. Kim H, Datta A, Talwar S, Saleem SN, Mondal D, Abdel-Mageed AB: Estradiol-ERß2 signaling axis confers growth and migration of CRPC cells through TMPRSS2-ETV5 gene fusion. Oncotarget 2016, 8(38):62820-62833.

16. Asselta R, Paraboschi EM, Mantovani A, Duga S: ACE2 and TMPRSS2 variants and expression as candidates to sex and country differences in COVID-19 severity in Italy. medRxiv 2020:2020.2003.2030.20047878.

17. Duga S, Asselta R, Lazzeri M, Guazzoni G, Azzolini E, Buffi N, Fasulo V, Persico F, Saita A, Hurle R et al: Impact of anti-androgenic therapies on COVID-19: an observational study in male population from a COVID-19 regional centre of Lombardy (Italy). Preprint at https://www.medrxiv.org/content/10.1101/2020.04.20.20068056v2.full.pdf. Accessed 21 September 2020

18. Gralinski LE, Ferris MT, Aylor DL, Whitmore AC, Green R, Frieman MB, Deming D, Menachery VD, Miller DR, Buus RJ et al: Genome Wide Identification of SARS-CoV Susceptibility Loci Using the Collaborative Cross. PLoS Genet 2015, 11(10):e1005504-e1005504. 
19. Zeng F, Dai C, Cai P, Wang J, Xu L, Li J, Hu G, Wang L: A comparison study of SARS-CoV-2 IgG antibody between male and female COVID-19 patients: a possible reason underlying different outcome between gender. medRxiv 2020:2020.2003.2026.20040709.

20. Klein SL, Flanagan KL: Sex differences in immune responses. Nature Reviews Immunology 2016, 16(10):626-638.

21. Hannah MF, Bajic VB, Klein SL: Sex differences in the recognition of and innate antiviral responses to Seoul virus in Norway rats. Brain Behav Immun 2008, 22(4):503-516.

22. Klein SL: Immune cells have sex and so should journal articles. Endocrinology 2012, 153(6):2544-2550.

23. Hewagama A, Patel D, Yarlagadda S, Strickland FM, Richardson BC: Stronger inflammatory/cytotoxic T-cell response in women identified by microarray analysis. Genes Immun 2009, 10(5):509-516.

24. Buskiewicz IA, Huber SA, Fairweather D: Sex hormone receptor expression in the immune system. In: Sex differences in physiology. edn.: Elsevier; 2016: 45-60.

25. Channappanavar R, Fett C, Mack M, Ten Eyck PP, Meyerholz DK, Perlman S: Sex-Based Differences in Susceptibility to Severe Acute Respiratory Syndrome Coronavirus Infection. J Immunol 2017, 198(10):4046-4053.

26. Parascandola M, Xiao L: Tobacco and the lung cancer epidemic in China. Transl Lung Cancer Res 2019, 8(Suppl 1):S21-S30.

27. Vardavas Cl, Nikitara K: COVID-19 and smoking: A systematic review of the evidence. Tob Induc Dis 2020, 18:20-20.

28. Cai G: Bulk and Single-Cell Transcriptomics Identify Tobacco-Use Disparity in Lung Gene Expression of ACE2, the Receptor of 2019-nCov. Preprints 2020.

29. Ali MA: Gender Dynamics and Socio-Cultural Determinants of Middle East Respiratory Syndrome Coronavirus (MERS-CoV) in Saudi Arabia. University of Toronto Medical Journal 2017, 94(1):32.

30. Zhu Z, Cai T, Fan L, Lou K, Hua X, Huang Z, Gao G: Clinical value of immune-inflammatory parameters to assess the severity of coronavirus disease 2019. International journal of infectious diseases : IJID : official publication of the International Society for Infectious Diseases 2020.

31. Zhou Y, Han T, Chen J, Hou C, Hua L, He S, Guo Y, Zhang S, Wang Y, Yuan J et al: Clinical and Autoimmune Characteristics of Severe and Critical Cases with COVID-19. Clinical and translational science 2020.

32. Zhou B, She J, Wang Y, Ma X: The clinical characteristics of myocardial injury in severe and very severe patients with 2019 novel coronavirus disease. The Journal of infection 2020.

33. Zheng Y, Xu H, Yang M, Zeng Y, Chen H, Liu R, Li Q, Zhang N, Wang D: Epidemiological characteristics and clinical features of 32 critical and 67 noncritical cases of COVID-19 in Chengdu. Journal of clinical virology : the official publication of the Pan American Society for Clinical Virology 2020, 127:104366104366.

34. Zheng S, Fan J, Yu F, Feng B, Lou B, Zou Q, Xie G, Lin S, Wang R, Yang X et al: Viral load dynamics and disease severity in patients infected with SARSCoV-2 in Zhejiang province, China, January-March 2020: retrospective cohort study. Bmj 2020, 369:m1443.

35. Zhao W, Zhong Z, Xie X, Yu Q, Liu J: Relation Between Chest CT Findings and Clinical Conditions of Coronavirus Disease (COVID-19) Pneumonia: A Multicenter Study. AJR American journal of roentgenology 2020:1-6.

36. Zhao W, Zhong Z, Xie X, Yu Q, Liu J: CT Scans of Patients with 2019 Novel Coronavirus (COVID-19) Pneumonia. Theranostics 2020, 10(10):4606-4613.

37. Zhang Y, Zheng L, Liu L, Zhao M, Xiao J, Zhao Q: Liver impairment in COVID-19 patients: a retrospective analysis of 115 cases from a single center in Wuhan city, China. Liver international : official journal of the International Association for the Study of the Liver 2020.

38. Zhang X, Cai H, Hu J, Lian J, Gu J, Zhang S, Ye C, Lu Y, Jin C, Yu G et al: Epidemiological, clinical characteristics of cases of SARS-CoV-2 infection with abnormal imaging findings. International journal of infectious diseases : IJID : official publication of the International Society for Infectious Diseases 2020.

39. Zhang R, Ouyang H, Fu L, Wang S, Han J, Huang K, Jia M, Song Q, Fu Z: CT features of SARS-CoV-2 pneumonia according to clinical presentation: a retrospective analysis of 120 consecutive patients from Wuhan city. European Radiology 2020.

40. Zhang J, Wang X, Jia X, Li J, Hu K, Chen G, Wei J, Gong Z, Zhou C, Yu H et al: Risk factors for disease severity, unimprovement, and mortality in COVID-19 patients in Wuhan, China. Clinical Microbiology and Infection 2020.

41. Zhang J, Liu P, Wang M, Wang J, Chen J, Yuan W, Li M, Xie Z, Dong W, Li H et al: The clinical data from 19 critically ill patients with coronavirus disease 2019: a single-centered, retrospective, observational study. Zeitschrift fur Gesundheitswissenschaften = Journal of public health 2020:1-4.

42. Zhang G, Hu C, Luo L, Fang F, Chen Y, Li J, Peng Z, Pan H: Clinical features and short-term outcomes of 221 patients with COVID-19 in Wuhan, China. Journal of Clinical Virology 2020, 127.

43. Yuan M, Yin W, Tao Z, Tan W, Hu Y: Association of radiologic findings with mortality of patients infected with 2019 novel coronavirus in Wuhan, China. PloS one 2020, 15(3):e0230548-e0230548.

44. Young BE, Ong SWX, Kalimuddin S, Low JG, Tan SY, Loh J, Ng O-T, Marimuthu K, Ang LW, Mak TM: Epidemiologic Features and Clinical Course of Patients Infected With SARS-CoV-2 in Singapore. JAMA 2020.

45. Yao Q, Wang P, Wang X, Qie G, Meng M, Tong X, Bai X, Ding M, Liu W, Liu K et al: Retrospective study of risk factors for severe SARS-Cov-2 infections in hospitalized adult patients. Polish archives of internal medicine 2020.

46. Yang F, Shi S, Zhu J, Shi J, Dai K, Chen X: Analysis of 92 deceased patients with COVID-19. Journal of medical virology 2020.

47. Yang A-P, Liu J-P, Tao W-Q, Li H-M: The diagnostic and predictive role of NLR, d-NLR and PLR in COVID-19 patients. International immunopharmacology 2020, 84:106504-106504.

48. Xu YH, Dong JH, An WM, Lv XY, Yin XP, Zhang JZ, Dong L, Ma X, Zhang HJ, Gao BL: Clinical and computed tomographic imaging features of novel coronavirus pneumonia caused by SARS-CoV-2. The Journal of infection 2020. 
49. Xu B, Fan C-Y, Wang A-L, Zou Y-L, Yu Y-H, He C, Xia W-G, Zhang J-X, Miao Q: Suppressed T cell-mediated immunity in patients with CoVID-19: A clinical retrospective study in Wuhan, China. The Journal of infection 2020.

50. Xiong Y, Sun D, Liu Y, Fan Y, Zhao L, Li X, Zhu W: Clinical and High-Resolution CT Features of the COVID-19 Infection: Comparison of the Initial and Followup Changes. Investigative radiology 2020.

51. Xie H, Zhao J, Lian N, Lin S, Xie Q, Zhuo H: Clinical characteristics of non-ICU hospitalized patients with coronavirus disease 2019 and liver injury: A retrospective study. Liver Int 2020.

52. Wang X, Liu W, Zhao J, Lu Y, Wang X, Yu C, Hu S, Shen N, Liu W, Sun Z et al: Clinical characteristics of 80 hospitalized frontline medical workers infected with COVID-19 in Wuhan, China. J Hosp Infect 2020.

53. Wang X, Fang J, Zhu Y, Chen L, Ding F, Zhou R, Ge L, Wang F, Chen Q, Zhang Y et al: Clinical characteristics of non-critically ill patients with novel coronavirus infection (COVID-19) in a Fangcang Hospital. Clinical microbiology and infection : the official publication of the European Society of Clinical Microbiology and Infectious Diseases 2020.

54. Wang R, Pan M, Zhang X, Fan X, Han M, Zhao F, Miao M, Xu J, Guan M, Deng X et al: Epidemiological and clinical features of 125 Hospitalized Patients with COVID-19 in Fuyang, Anhui, China. International journal of infectious diseases : IJID : official publication of the International Society for Infectious Diseases 2020.

55. Wang L, He W, Yu X, Hu D, Bao M, Liu H, Zhou J, Jiang H: Coronavirus disease 2019 in elderly patients: Characteristics and prognostic factors based on 4week follow-up. The Journal of infection 2020.

56. Wang J, Xu Z, Feng R, An Y, Ao W, Gao Y, Wang X, Xie Z: CT characteristics of patients infected with 2019 novel coronavirus: association with clinical type. Clinical Radiology 2020.

57. Wang G, Chen W, Jin X, Chen Y-P: Description of COVID-19 cases along with the measures taken on prevention and control in Zhejiang, China. Journal of medical virology 2020.

58. Sun C, Zhang XB, Dai Y, Xu XZ, Zhao J: Clinical analysis of 150 cases of 2019 novel coronavirus infection in Nanyang City, Henan Province. Zhonghua jie he he hu xi za zhi = Zhonghua jiehe he huxi zazhi = Chinese journal of tuberculosis and respiratory diseases 2020, 43(0):E042-E042.

59. Simonnet A, Chetboun M, Poissy J, Raverdy V, Noulette J, Duhamel A, Labreuche J, Mathieu D, Pattou F, Jourdain M: High prevalence of obesity in severe acute respiratory syndrome coronavirus-2 (SARS-CoV-2) requiring invasive mechanical ventilation. Obesity (Silver Spring, Md) 2020.

60. Richardson S, Hirsch JS, Narasimhan M, Crawford JM, McGinn T, Davidson KW, Barnaby DP, Becker LB, Chelico JD, Cohen SL et al: Presenting Characteristics, Comorbidities, and Outcomes Among 5700 Patients Hospitalized With COVID-19 in the New York City Area. Jama 2020.

61. Qian ZP, Mei X, Zhang YY, Zou Y, Zhang ZG, Zhu H, Guo HY, Liu Y, Ling Y, Zhang XY et al: Analysis of baseline liver biochemical parameters in 324 cases with novel coronavirus pneumonia in Shanghai area. Zhonghua gan zang bing za zhi = Zhonghua ganzangbing zazhi = Chinese journal of hepatology 2020, 28(0):E005-E005.

62. Niu S, Tian S, Lou J, Kang X, Zhang L, Lian H, Zhang J: Clinical characteristics of older patients infected with COVID-19: A descriptive study. Archives of Gerontology and Geriatrics 2020, 89.

63. Lyu P, Liu X, Zhang R, Shi L, Gao J: The performance of chest CT in evaluating the clinical severity of COVID-19 pneumonia: identifying critical cases based on CT characteristics. Investigative radiology 2020.

64. Liu Y, Du X, Chen J, Jin Y, Peng L, Wang HHX, Luo M, Chen L, Zhao Y: Neutrophil-to-lymphocyte ratio as an independent risk factor for mortality in hospitalized patients with COVID-19. The Journal of infection 2020.

65. Liu KC, Xu P, Lv WF, Qiu XH, Yao JL, Gu JF, Wei W: CT manifestations of coronavirus disease-2019: A retrospective analysis of 73 cases by disease severity. European Journal of Radiology 2020, 126 (no pagination)(108941).

66. Liu K, Chen Y, Lin R, Han K: Clinical feature of COVID-19 in elderly patients: a comparison with young and middle-aged patients. The Journal of infection 2020.

67. Liang W-H, Guan W-J, Li C-C, Li Y-M, Liang H-R, Zhao Y, Liu X-Q, Sang L, Chen R-C, Tang C-L et al: Clinical characteristics and outcomes of hospitalised patients with COVID-19 treated in Hubei (epicenter) and outside Hubei (non-epicenter): A Nationwide Analysis of China. The European respiratory journal 2020.

68. Li X, Xu S, Yu M, Wang K, Tao Y, Zhou Y, Shi J, Zhou M, Wu B, Yang Z et al: Risk factors for severity and mortality in adult COVID-19 inpatients in Wuhan. The Journal of allergy and clinical immunology 2020.

69. Li K, Wu J, Wu F, Guo D, Chen L, Fang Z, Li C: The Clinical and Chest CT Features Associated with Severe and Critical COVID-19 Pneumonia. Investigative radiology 2020.

70. Lei Z, Cao H, Jie Y, Huang Z, Guo X, Chen J, Peng L, Cao H, Dai X, Liu J et al: A cross-sectional comparison of epidemiological and clinical features of patients with coronavirus disease (COVID-19) in Wuhan and outside Wuhan, China. Travel medicine and infectious disease 2020:101664.

71. He GH, Rong ZH, Hu JX, Liu T, Xiao JP, Guo LC, Zeng WL, Zhu ZH, Gong DX, Yin LH et al: Comparison of two epidemic patterns of COVID-19 and evaluation of prevention and control effectiveness: an analysis based on Guangzhou and Wenzhou. Zhonghua liu xing bing xue za zhi = Zhonghua liuxingbingxue zazhi 2020, 41:E035.

72. Han C, Duan C, Zhang S, Spiegel B, Shi H, Wang W, Zhang L, Lin R, Liu J, Ding Z et al: Digestive Symptoms in COVID-19 Patients With Mild Disease Severity: Clinical Presentation, Stool Viral RNA Testing, and Outcomes. The American journal of gastroenterology 2020.

73. Goyal P, Choi JJ, Pinheiro LC, Schenck EJ, Chen R, Jabri A, Satlin MJ, Campion TR, Nahid M, Ringel JB et al: Clinical Characteristics of Covid-19 in New York City. 2020. 
74. Gou FX, Zhang XS, Yao JX, Yu DS, Wei KF, Zhang H, Yang XT, Yang JJ, Liu HX, Cheng Y et al: Epidemiological characteristics of COVID-19 in Gansu province. Zhonghua liu xing bing xue za zhi = Zhonghua liuxingbingxue zazhi 2020, 41:E032.

75. Fan Z, Chen L, Li J, Cheng X, Jingmao Y, Tian C, Zhang Y, Huang S, Liu Z, Cheng J: Clinical Features of COVID-19-Related Liver Damage. Clinical gastroenterology and hepatology : the official clinical practice journal of the American Gastroenterological Association 2020.

76. Du Y, Tu L, Zhu P, Mu M, Wang R, Yang P, Wang X, Hu C, Ping R, Hu P et al: Clinical Features of 85 Fatal Cases of COVID-19 from Wuhan: A Retrospective Observational Study. American journal of respiratory and critical care medicine 2020.

77. Dong XC, Li JM, Bai JY, Liu ZQ, Zhou PH, Gao L, Li XY, Zhang Y: Epidemiological characteristics of confirmed COVID-19 cases in Tianjin. Zhonghua liu xing bing xue za zhi = Zhonghua liuxingbingxue zazhi 2020, 41(5):638-642.

78. Dai H, Zhang X, Xia J, Zhang T, Shang Y, Huang R, Liu R, Wang D, Li M, Wu J et al: High-resolution Chest CT Features and Clinical Characteristics of Patients Infected with COVID-19 in Jiangsu, China. International journal of infectious diseases : IJID : official publication of the International Society for Infectious Diseases 2020.

79. Chu J, Yang N, Wei Y, Yue H, Zhang F, Zhao J, He L, Sheng G, Chen P, Li G et al: Clinical Characteristics of 54 medical staff with COVID-19: A retrospective study in a single center in Wuhan, China. Journal of medical virology 2020.

80. Chen T, Dai Z, Mo P, Li X, Ma Z, Song S, Chen X, Luo M, Liang K, Gao S et al: Clinical characteristics and outcomes of older patients with coronavirus disease 2019 (COVID-19) in Wuhan, China (2019): a single-centered, retrospective study. The journals of gerontology Series A, Biological sciences and medical sciences 2020

81. Cao J, Tu WJ, Cheng W, Yu L, Liu YK, Hu X, Liu Q: Clinical Features and Short-term Outcomes of 102 Patients with Corona Virus Disease 2019 in Wuhan, China. Clinical infectious diseases : an official publication of the Infectious Diseases Society of America 2020.

82. Cai Q, Huang D, Ou P, Yu H, Zhu Z, Xia Z, Su Y, Ma Z, Zhang Y, Li Z et al: COVID-19 in a designated infectious diseases hospital outside Hubei Province, China. Allergy 2020.

83. Arentz M, Yim E, Klaff L, Lokhandwala S, Riedo FX, Chong M, Lee M: Characteristics and Outcomes of 21 Critically III Patients with COVID-19 in Washington State. JAMA - Journal of the American Medical Association 2020.

84. Novel Coronavirus Pneumonia Emergency Response Epidemiology T: The epidemiological characteristics of an outbreak of 2019 novel coronavirus diseases (COVID-19) in China. Zhonghua liu xing bing xue za zhi = Zhonghua liuxingbingxue zazhi 2020, 41(2):145-151.

85. Fu S, Fu X, Song Y, Li M, Pan P-h, Tang T, Zhang C, Jiang T, Tan D, Fan X et al: Virologic and clinical characteristics for prognosis of severe CoVID-19: a retrospective observational study in Wuhan, China. 2020:2020.2004.2003.20051763.

86. Fei J, Fu L, Li Y, Xiang H-X, Xiang Y, Li M-D, Liu F-F, Xu D-x, Zhao H: Reduction of lymphocyte at early stage elevates severity and death risk of COVID-19 patients: a hospital-based case-cohort study. 2020:2020.2004.2002.20050955.

87. Fu L, Fei J, Xu S, Xiang H-X, Xiang Y, Tan Z-X, Li M-D, Liu F-F, Li Y, Han M-F et al: Acute liver injury and its association with death risk of patients with COVID-19: a hospital-based prospective case-cohort study. 2020:2020.2004.2002.20050997.

88. Mazumder A, Arora M, Bharadiya V, Berry P, Agarwal M, Gupta M, Parameswaran GG, Behera P: Study of Epidemiological Characteristics and In-silico Analysis of the Effect of Interventions in the SARS-CoV-2 Epidemic in India. 2020:2020.2004.2005.20053884.

89. Tan L, Kang X, Ji X, Wang Q, li Y, Wang Q, Miao H: Validation of reported risk factors for disease classification and prognosis in COVID-19: a descriptive and retrospective study. Preprint at https://www.medrxiv.org/content/10.1101/2020.04.05.20053769v1.full.pdf. Accessed 07 April 2020

90. Xiao G, Hu H, Wu F, Sha T, Huang Q, Li H, Han J, Song W, Chen Z, Zeng Z: Acute kidney injury in patients hospitalized with COVID-19 in Wuhan, China: A single-center retrospective observational study. Preprint at https://www.medrxiv.org/content/10.1101/2020.04.06.20055194v1.full.pdf. Accessed 08 April 2020

91. wang Z, Ji JS, Liu Y, Liu R, Zha Y, Chang X, Zhang L, Zhang Y, Zeng J, Dong T et al: Survival analysis of hospital length of stay of novel coronavirus (COVID-19) pneumonia patients in Sichuan, China. Preprint at https://www.medrxiv.org/content/10.1101/2020.04.07.20057299v1.full.pdf. Accessed 10 April 2020

92. Petrilli CM, Jones SA, Yang J, Rajagopalan H, O'Donnell LF, Chernyak Y, Tobin K, Cerfolio RJ, Francois F, Horwitz LI: Factors associated with hospitalization and critical illness among 4,103 patients with COVID-19 disease in New York City. 2020:2020.2004.2008.20057794.

93. Chen X, Zhang Y, Zhu B, Zeng J, Hong W, He X, Chen J, Zheng H, Qiu S, Deng Y et al: Associations of clinical characteristics and antiviral drugs with viral RNA clearance in patients with COVID-19 in Guangzhou, China: a retrospective cohort study. Preprint at https://www.medrxiv.org/content/10.1101/2020.04.09.20058941v1.full.pdf. Accessed 14 April 2020

94. Jiang X, Tao J, Wu H, Wang Y, Zhao W, Zhou M, Huang J, You Q, Meng H, Zhu F et al: Clinical features and management of severe COVID-19: A retrospective study in Wuxi, Jiangsu Province, China. Preprint at https://www.medrxiv.org/content/10.1101/2020.04.10.20060335v1.full.pdf. Accessed 14 April 2020

95. Guo A-X, Cui J-J, OuYang Q-Y, He L, Guo C-X, Yin J-Y: The clinical characteristics and mortal causes analysis of COVID-19 death patients. Preprint at https://www.medrxiv.org/content/10.1101/2020.04.12.20062380v1.full.pdf. Accessed 15 April 2020

96. Zheng Y, Sun L-j, Xu M, Pan J, Zhang Y-t, Fang X-I, Fang Q, Cai H-l: Clinical characteristics of 34 COVID-19 patients admitted to intensive care unit in Hangzhou, China. Journal of Zhejiang University-SCIENCE B 2020, 21(5):378-387.

97. Shao Z, Feng Y, Zhong L, Xie Q, Lei M, Liu Z, Wang C, Ji J, Liu H, Gu Z et al: Clinical efficacy of intravenous immunoglobulin therapy in critical ill patients with COVID-19: a multicenter retrospective cohort study. Clin Trans/ Immunology 2020, 9(10):e1192.

98. Lewnard JA, Liu VX, Jackson ML, Schmidt MA, Jewell BL, Flores JP, Jentz C, Northrup GR, Mahmud A, Reingold AL et al: Incidence, clinical outcomes, and transmission dynamics of hospitalized 2019 coronavirus disease among 9,596,321 individuals residing in California and Washington, United States: a

Page $17 / 24$ 
prospective cohort study. Preprint at https://www.medrxiv.org/content/10.1101/2020.04.12.20062943v1.full.pdf. Accessed 16 April 2020

99. Zeng X, Fan H, Lu D, Huang F, Meng X, Li Z, Tang M, Zhang J, Liu N, Liu Z et al: Association between ABO blood groups and clinical outcome of coronavirus disease 2019: Evidence from two cohorts. Preprint at https://www.medrxiv.org/content/10.1101/2020.04.15.20063107v1.full.pdf. Accessed 17 April 2020

100. Cummings MJ, Baldwin MR, Abrams D, Jacobson SD, Meyer BJ, Balough EM, Aaron JG, Claassen J, Rabbani LE, Hastie J et al: Epidemiology, clinical course, and outcomes of critically ill adults with COVID-19 in New York City: a prospective cohort study. 2020:2020.2004.2015.20067157.

101. Argenziano MG, Bruce SL, Slater CL, Tiao JR, Baldwin MR, Barr RG, Chang BP, Chau KH, Choi JJ, Gavin N et al: Characterization and Clinical Course of 1000 Patients with COVID-19 in New York: retrospective case series. Preprint at https://www.medrxiv.org/content/10.1101/2020.04.20.20072116v2.full.pdf. Accessed 22 April 2020

102. Shi P, Ren G, Yang J, Li Z, Deng S, Li M, Wang S, Xu X, Chen F, Li Y et al: Clinical characteristics of imported and second-generation COVID-19 cases outside Wuhan, China: A multicenter retrospective study. Preprint at https://www.medrxiv.org/content/10.1101/2020.04.19.20071472v1.full.pdf. Accessed 23 April 2020

103. Kong X, Zheng K, Tang M, Kong F, Zhou J, Diao L, Wu S, Jiao P, Su T, Dong Y: Prevalence and Factors Associated with Depression and Anxiety of Hospitalized Patients with COVID-19. Preprint at https://www.medrxiv.org/content/10.1101/2020.03.24.20043075v2.full.pdf Accessed 05 April 2020

104. Huang H, Cai S, Li Y, Li Y, Fan Y, Li L, Lei C, Tang X, Hu F, Li F et al: Prognostic Factors for COVID-19 Pneumonia Progression to Severe Symptoms Based on Earlier Clinical Features: A Retrospective Analysis. Front Med (Lausanne) 2020, 7:557453.

105. Zhou F, Yu X, Tong X, Zhang R: Clinical features and outcomes of 197 adult discharged patients with COVID-19 in Yichang, Hubei. Preprint at https://www.medrxiv.org/content/10.1101/2020.03.26.20041426v2.full.pdf. Accessed 05 April 2020

106. Xie J, Hungerford D, Chen H, Abrams ST, Li S, Wang G, Wang Y, Kang H, Bonnett L, Zheng R et al: Development and external validation of a prognostic multivariable model on admission for hospitalized patients with COVID-19. Preprint at https://www.medrxiv.org/content/10.1101/2020.03.28.20045997v2.full.pdf. Accessed 07 April 2020

107. Zeng L, Li J, Liao M, Hua R, Huang P, Zhang M, Zhang Y, Shi Q, Xia Z, Ning X et al: Risk assessment of progression to severe conditions for patients with COVID-19 pneumonia: a single-center retrospective study. Preprint at https://www.medrxiv.org/content/10.1101/2020.03.25.20043166v1.full.pdf. Accessed 30 March 2020

108. Chen X, Jiang Q, Ma Z, Ling J, Hu W, Cao Q, Mo P, Yang R, Gao S, Gui X et al: Clinical Characteristics of Hospitalized Patients with SARS-CoV-2 and Hepatitis B virus Co-infection. medRxiv 2020:2020.2003.2023.20040733.

109. Chen X, Jiang Q, Ma Z, Ling J, Hu W, Cao Q, Mo P, Yao L, Yang R, Gao S et al: Clinical Characteristics of Hospitalized Patients with SARS-CoV-2 and Hepatitis B Virus Co-infection. Virol Sin 2020.

110. Li K, Chen D, Chen S, Feng Y, Chang C, Wang Z, Wang N, Zhen G: Predictors of fatality including radiographic findings in adults with COVID-19. Respiratory Research 2020, 21(1):146.

111. Yan D, Liu X-Y, Zhu Y-n, Huang L, Dan B-t, Zhang G-j, Gao Y-h: Factors associated with prolonged viral shedding and impact of lopinavir/ritonavir treatment in hospitalised non-critically ill patients with SARS-CoV-2 infection. 2020, 56(1):2000799.

112. Wang Z, Li H, Li J, Yang C, Guo X, Hu Z, Chen Z, Wang S, Liu J, Li WJSeEP: Elevated Serum IgM Levels Indicate Poor Outcome in Patients with Coronavirus Disease 2019 Pneumonia: A Retrospective Case-Control Study.

113. Liao X, Chen H, Wang B, Li Z, Zhang Z, Li W, Liang Z, Tang J, Wang J, Shi R et al: Critical Care for Severe COVID-19: A Population-based Study from a Province with Low Case-fatality Rate in China. Preprint at https://www.medrxiv.org/content/10.1101/2020.03.22.20041277v4.full.pdf. Accessed 21 April 2020

114. Zhang Y, Cui Y, Shen M, Zhang J, Liu B, Dai M, Chen L, Han D, Fan Y, Zeng Y et al: Comorbid Diabetes Mellitus was Associated with Poorer Prognosis in Patients with COVID-19: A Retrospective Cohort Study. Preprint at https://www.medrxiv.org/content/10.1101/2020.03.24.20042358v1.full.pdf. Accessed 26 March 2020

115. Nie S, Zhao X, Zhao K, Zhang Z, Zhang Z, Zhang Z: Metabolic disturbances and inflammatory dysfunction predict severity of coronavirus disease 2019 (COVID-19): a retrospective study. Preprint at https://www.medrxiv.org/content/10.1101/2020.03.24.20042283v1.full.pdf. Accessed 26 March 2020

116. Xu S, Fu L, Fei J, Xiang H-X, Xiang Y, Tan Z-X, Li M-D, Liu F-F, Li Y, Han M-F et al: Acute kidney injury at early stage as a negative prognostic indicator of patients with COVID-19: a hospital-based retrospective analysis. Preprint at https://www.medrxiv.org/content/10.1101/2020.03.24.20042408v1.full.pdf. Accessed 26 March 2020

117. Hu L, Chen S, Fu Y, Gao Z, Long H, Ren H-w, Zuo Y, Li H, Wang J, Xv Q-b et al: Risk Factors Associated with Clinical Outcomes in 323 COVID-19 Patients in Wuhan, China. Preprint at https://www.medrxiv.org/content/10.1101/2020.03.25.20037721v2.full.pdf Accessed 30 March 2020

118. Wen Y, Wei L, Li Y, Tang X, Feng S, Leung K, Wu X, Pan X-F, Chen C, Xia J et al: Epidemiological and clinical characteristics of COVID-19 in Shenzhen, the largest migrant city of China. Preprint at https://www.medrxiv.org/content/10.1101/2020.03.22.20035246v1.full.pdf. Accessed 23 March 2020

119. Yan S, Song X, Lin F, Zhu H, Wang X, Li M, Ruan J, Lin C, Liu X, Wu Q et al: Clinical Characteristics of Coronavirus Disease 2019 in Hainan, China. Preprint at https://www.medrxiv.org/content/10.1101/2020.03.19.20038539v1.full.pdf. Accessed 23 March 2020

120. Effect of Continuous Renal Replacement Therapy on Patients with Acute Renal Failure in ICU \%J Chinese Journal of Bases and Clinics in General Surgery. 2004.

121. Coronavirus Disease-19: The First 7,755 Cases in the Republic of Korea. Osong Public Health Res Perspect 2020, 11(2):85-90.

122. Coronavirus Disease-19: The First 7,755 Cases in the Republic of Korea. Osong Public Health Res Perspect 2020, 11(2):85-90. 
123. Zhao W, Yu S, Zha X, Wang N, Pang Q, Li T, Li A: Clinical characteristics and durations of hospitalized patients with COVID-19 in Beijing: a retrospective cohort study. Preprint at https://www.medrxiv.org/content/10.1101/2020.03.13.20035436v2.full.pdf. Accessed 30 March 2020

124. Fu L, Fei J, Xiang H-X, Xiang Y, Tan Z-X, Li M-D, Liu F-F, Liu H-Y, Zheng L, Li Y et al: Influence factors of death risk among COVID-19 patients in Wuhan, China: a hospital-based case-cohort study. Preprint at https://www.medrxiv.org/content/10.1101/2020.03.13.20035329v1.full.pdf. Accessed 16 March 2020

125. Chen L, Deng C, Chen X, Zhang X, Chen B, Yu H, Qin Y, Xiao K, Zhang H, Sun X: Ocular manifestations and clinical characteristics of 535 cases of COVID19 in Wuhan, China: a cross-sectional study. Acta Ophthalmol 2020.

126. liu y, Li J, liu D, Song H, chen C, Iv M, pei X, Hu Z: Clinical features and outcomes of 2019 novel coronavirus-infected patients with cardiac injury. Preprint at https://www.medrxiv.org/content/10.1101/2020.03.11.20030957v1.full.pdf. Accessed 16 March 2020

127. Zhang L, Huang B, Xia H, Fan H, Zhu M, Zhu L, Zhang H, Tao X, Cheng S, Chen J: Retrospective analysis of clinical features in 134 coronavirus disease 2019 cases. Epidemiology and infection 2020, 148:e199.

128. Wu Y, Guo W, Liu H, Qi B, Liang K, Xu H, Peng Z, Xiao S-Y: Clinical outcomes of 402 patients with COVID-2019 from a single center in Wuhan, China. Journal of medical virology 2020, 92(11):2751-2757.

129. Xu Y, Li Y-r, Zeng Q, Lu Z-b, Li Y-z, Wu W, Dong S-y, Huang G, Wang X-h: Clinical Characteristics of SARS-CoV-2 Pneumonia Compared to Controls in Chinese Han Population. Preprint at https://www.medrxiv.org/content/10.1101/2020.03.08.20031658v1.full.pdf. Accessed 10 March 2020

130. Shi Q, Zhao K, Yu J, Jiang F, Feng J, Zhao K, Zhang X, Chen X, Hu P, Hong Y et al: Clinical characteristics of 101 COVID-19 nonsurvivors in Wuhan, China: a retrospective study. Preprint at https://www.medrxiv.org/content/10.1101/2020.03.04.20031039v4.full.pdf. Accessed 20 May 2020

131. Xu Y, Xu Z, Liu X, Cai L, Zheng H, Huang Y, Zhou L, Huang L, Lin Y, Deng L et al: Clinical findings in critical ill patients infected with SARS-Cov-2 in Guangdong Province, China: a multi-center, retrospective, observational study. Preprint at https://www.medrxiv.org/content/10.1101/2020.03.03.20030668v1.full.pdf. Accessed 06 March 2020

132. Zhang G, Hu C, Luo L, Fang F, Chen Y, Li J, Peng Z, Pan H: Clinical features and short-term outcomes of 221 patients with COVID-19 in Wuhan, China. Journal of clinical virology : the official publication of the Pan American Society for Clinical Virology 2020, 127:104364.

133. Cao M, Zhang D, Wang Y, Lu Y, Zhu X, Li Y, Xue H, Lin Y, Zhang M, Sun Y et al: Clinical Features of Patients Infected with the 2019 Novel Coronavirus (COVID-19) in Shanghai, China. Preprint at https://www.medrxiv.org/content/10.1101/2020.03.04.20030395v1.full.pdf. Accessed 06 Mar 2020

134. Chen X, Zheng F, Qing Y, Ding S, Yang D, Lei C, Yin Z, Zhou X, Jiang D, Zuo Q et al: Epidemiological and clinical features of 291 cases with coronavirus disease 2019 in areas adjacent to Hubei, China: a double-center observational study. Preprint at https://www.medrxiv.org/content/10.1101/2020.03.03.20030353v1.full.pdf. Accessed 06 March 2020

135. Wang Y, Zhou Y, Yang Z, Xia D, Hu Y, Geng S: Clinical Characteristics of Patients with Severe Pneumonia Caused by the SARS-CoV-2 in Wuhan, China. Respiration 2020, 99(8):649-657.

136. Qi D, Yan X, Tang X, Peng J, Yu Q, Feng L, Yuan G, Zhang A, Chen Y, Yuan J et al: Epidemiological and clinical features of 2019-nCoV acute respiratory disease cases in Chongqing municipality, China: a retrospective, descriptive, multiple-center study. Preprint at https://www.medrxiv.org/content/10.1101/2020.03.01.20029397v1.full.pdf Accessed 03 March 2020

137. Xu L, Yuan J, Zhang Y, Zhang G, Lu F, Su J, Qu J: Highland of COVID-19 outside Hubei: epidemic characteristics, control and projections of Wenzhou, China. Preprint at https://www.medrxiv.org/content/10.1101/2020.02.25.20024398v1.full.pdf. Accessed 29 February 2020

138. Zhang B, Zhou X, Qiu Y, Song Y, Feng F, Feng J, Song Q, Jia Q, Wang J: Clinical characteristics of 82 cases of death from COVID-19. PloS one 2020, 15(7):e0235458.

139. Liang Y, Liang J, Zhou Q, Li X, Lin F, Deng Z, Zhang B, Li L, Wang X, Zhu H et al: Prevalence and clinical features of 2019 novel coronavirus disease (COVID-19) in the Fever Clinic of a teaching hospital in Beijing: a single-center, retrospective study Preprint at https://www.medrxiv.org/content/10.1101/2020.02.25.20027763v2.full.pdf Accessed 28 February 2020

140. Fan Z, Chen L, Li J, Cheng X, Yang J, Tian C, Zhang Y, Huang S, Liu Z, Cheng J: Clinical Features of COVID-19-Related Liver Functional Abnormality. Clinical Gastroenterology and Hepatology 2020, 18(7):1561-1566.

141. Cao W: Clinical features and laboratory inspection of novel coronavirus pneumonia (COVID-19) in Xiangyang, Hubei. Preprint at https://www.medrxiv.org/content/10.1101/2020.02.23.20026963v1.full.pdf. Accessed 25 February 2020

142. Jin J-M, Bai P, He W, Liu S, Wu F, Liu X-F, Han D-M, Yang J-KJm: Higher severity and mortality in male patients with COVID-19 independent of age and susceptibility. Preprint at https://www.medrxiv.org/content/medrxiv/early/2020/02/25/2020.02.23.20026864.full.pdf. Accessed 25 February 2020

143. Li X, Wang L, Yan S, Yang F, Xiang L, Zhu J, Shen B, Gong Z: Clinical characteristics of 25 death cases with COVID-19: A retrospective review of medical records in a single medical center, Wuhan, China. International journal of infectious diseases : IJID : official publication of the International Society for Infectious Diseases 2020, 94:128-132.

144. Wang L, Li X, Chen H, Yan S, Li Y, Li D, Gong Z: SARS-CoV-2 infection does not significantly cause acute renal injury: an analysis of 116 hospitalized patients with COVID-19 in a single hospital, Wuhan, China. Preprint at https://www.medrxiv.org/content/10.1101/2020.02.19.20025288v1.full.pdf. Accessed 23 February 2020

145. Ai J, Chen J, Wang Y, Liu X, Fan W, Qu G, Zhang M, Pei SP, Tang B, Yuan S et al: The cross-sectional study of hospitalized coronavirus disease 2019 patients in Xiangyang, Hubei province. Preprint at https://www.medrxiv.org/content/10.1101/2020.02.19.20025023v1.full.pdf. Accessed 23 Feb 2020

146. Lei L, Jian-ya G: Clinical characteristics of 51 patients discharged from hospital with COVID-19 in Chongqing,China Preprint at ttps://www.medrxiv.org/content/10.1101/2020.02.20.20025536v1.full.pdf. Accessed 23 February 2020 
147. Liu Y, Sun W, Li J, Chen L, Wang Y, Zhang L, Yu L: Clinical features and progression of acute respiratory distress syndrome in coronavirus disease 2019. Preprint at https://www.medrxiv.org/content/10.1101/2020.02.17.20024166v3.full.pdf. Accessed 27 February 2020

148. Wu C, Chen X, Cai Y, Xia J, Zhou X, Xu S, Huang H, Zhang L, Zhou X, Du C et al: Risk Factors Associated With Acute Respiratory Distress Syndrome and Death in Patients With Coronavirus Disease 2019 Pneumonia in Wuhan, China. JAMA internal medicine 2020.

149. Shi H, Han X, Jiang N, Cao Y, Alwalid O, Gu J, Fan Y, Zheng C: Radiological findings from 81 patients with COVID-19 pneumonia in Wuhan, China: a descriptive study. The Lancet Infectious diseases 2020, 20(4):425-434.

150. Pan Y, Guan H, Zhou S, Wang Y, Li Q, Zhu T, Hu Q, Xia L: Initial CT findings and temporal changes in patients with the novel coronavirus pneumonia (2019nCoV): a study of 63 patients in Wuhan, China. European Radiology 2020.

151. Wang K, Kang S, Tian R, Zhang X, Wang Y: Imaging manifestations and diagnostic value of chest CT of coronavirus disease 2019 (COVID-19) in the Xiaogan area. Clinical Radiology 2020.

152. Shi Y, Yu X, Zhao H, Wang H, Zhao R, Sheng J: Host susceptibility to severe COVID-19 and establishment of a host risk score: findings of 487 cases outside Wuhan. Critical care (London, England) 2020, 24(1):108.

153. McMichael TM, Currie DW, Clark S, Pogosjans S, Kay M, Schwartz NG, Lewis J, Baer A, Kawakami V, Lukoff MD et al: Epidemiology of Covid-19 in a LongTerm Care Facility in King County, Washington. The New England journal of medicine 2020.

154. Jin X, Lian JS, Hu JH, Gao J, Zheng L, Zhang YM, Hao SR, Jia HY, Cai H, Zhang XL et al: Epidemiological, clinical and virological characteristics of 74 cases of coronavirus-infected disease 2019 (COVID-19) with gastrointestinal symptoms. Gut 2020.

155. Chen N, Zhou M, Dong X, Qu J, Gong F, Han Y, Qiu Y, Wang J, Liu Y, Wei Y et al: Epidemiological and clinical characteristics of 99 cases of 2019 novel coronavirus pneumonia in Wuhan, China: a descriptive study. Lancet 2020, 395(10223):507-513.

156. Zhou F, Yu T, Du R, Fan G, Liu Y, Liu Z, Xiang J, Wang Y, Song B, Gu X et al: Clinical course and risk factors for mortality of adult inpatients with COVID-19 in Wuhan, China: a retrospective cohort study. Lancet 2020.

157. Zhang JJ, Dong X, Cao YY, Yuan YD, Yang YB, Yan YQ, Akdis CA, Gao YD: Clinical characteristics of 140 patients infected with SARS-CoV-2 in Wuhan, China. Allergy: European Journal of Allergy and Clinical Immunology 2020.

158. Zhang G, Zhang J, Wang B, Zhu X, Wang Q, Qiu S: Analysis of clinical characteristics and laboratory findings of 95 cases of 2019 novel coronavirus pneumonia in Wuhan, China: a retrospective analysis. Respiratory research 2020, 21(1):74-74.

159. Yang W, Cao Q, Qin L, Wang X, Cheng Z, Pan A, Dai J, Sun Q, Zhao F, Qu J et al: Clinical characteristics and imaging manifestations of the 2019 novel coronavirus disease (COVID-19):A multi-center study in Wenzhou city, Zhejiang, China. The Journal of infection 2020, 80(4):388-393.

160. Xu XW, Wu XX, Jiang XG, Xu KJ, Ying LJ, Ma CL, Li SB, Wang HY, Zhang S, Gao HN et al: Clinical findings in a group of patients infected with the 2019 novel coronavirus (SARS-Cov-2) outside of Wuhan, China: retrospective case series. Bmj 2020, 368:m606.

161. Wu J, Liu J, Zhao X, Liu C, Wang W, Wang D, Xu W, Zhang C, Yu J, Jiang B et al: Clinical Characteristics of Imported Cases of COVID-19 in Jiangsu Province: A Multicenter Descriptive Study. Clinical infectious diseases : an official publication of the Infectious Diseases Society of America 2020.

162. Wang D, Hu B, Hu C, Zhu F, Liu X, Zhang J, Wang B, Xiang H, Cheng Z, Xiong Y et al: Clinical Characteristics of 138 Hospitalized Patients With 2019 Novel Coronavirus-Infected Pneumonia in Wuhan, China. Jama 2020.

163. Wan S, Xiang Y, Fang W, Zheng Y, Li B, Hu Y, Lang C, Huang D, Sun Q, Xiong Y et al: Clinical Features and Treatment of COVID-19 Patients in Northeast Chongqing. Journal of medical virology 2020.

164. Tian S, Hu N, Lou J, Chen K, Kang X, Xiang Z, Chen H, Wang D, Liu N, Liu D et al: Characteristics of COVID-19 infection in Beijing. The Journal of infection 2020.

165. Qian GQ, Yang NB, Ding F, Ma AHY, Shen YF, Shi CW, Lian X, Chu JG, Chen L, Wang ZY et al: Epidemiologic and Clinical Characteristics of 91 Hospitalized Patients with COVID-19 in Zhejiang, China: A retrospective, multi-centre case series. QJM : monthly journal of the Association of Physicians $2020,17$.

166. Kui L, Fang YY, Deng Y, Liu W, Wang MF, Ma JP, Xiao W, Wang YN, Zhong MH, Li CH et al: Clinical characteristics of novel coronavirus cases in tertiary hospitals in Hubei Province. Chinese medical journal 2020.

167. Lian J, Jin X, Hao S, Cai H, Zhang S, Zheng L, Jia H, Hu J, Gao J, Zhang Y et al: Analysis of Epidemiological and Clinical features in older patients with Corona Virus Disease 2019 (COVID-19) out of Wuhan. Clinical infectious diseases : an official publication of the Infectious Diseases Society of America 2020, 25.

168. Li Q, Guan X, Wu P, Wang X, Zhou L, Tong Y, Ren R, Leung KSM, Lau EHY, Wong JY et al: Early Transmission Dynamics in Wuhan, China, of Novel Coronavirus-Infected Pneumonia. N Engl J Med 2020.

169. Lescure FX, Bouadma L, Nguyen D, Parisey M, Wicky PH, Behillil S, Gaymard A, Bouscambert-Duchamp M, Donati F, Le Hingrat Q et al: Clinical and virological data of the first cases of COVID-19 in Europe: a case series. The Lancet Infectious diseases 2020.

170. Huang Y, Tu M, Wang S, Chen S, Zhou W, Chen D, Zhou L, Wang M, Zhao Y, Zeng W et al: Clinical characteristics of laboratory confirmed positive cases of SARS-CoV-2 infection in Wuhan, China: A retrospective single center analysis. Travel medicine and infectious disease 2020:101606.

171. Huang C, Wang Y, Li X, Ren L, Zhao J, Hu Y, Zhang L, Fan G, Xu J, Gu X et al: Clinical features of patients infected with 2019 novel coronavirus in Wuhan, China. Lancet 2020, 395(10223):497-506.

172. Guan WJ, Ni ZY, Hu Y, Liang WH, Ou CQ, He JX, Liu L, Shan H, Lei CL, Hui DSC et al: Clinical Characteristics of Coronavirus Disease 2019 in China. N Engl J Med 2020.

173. Easom N, Moss P, Barlow G, Samson A, Taynton T, Adams K, Ivan M, Burns P, Gajee K, Eastick K et al: 68 Consecutive patients assessed for COVID-19 infection; experience from a UK regional infectious disease unit. Influenza and other respiratory viruses 2020. 
174. Deng Y, Liu W, Liu K, Fang YY, Shang J, Zhou L, Wang K, Leng F, Wei S, Chen L et al: Clinical characteristics of fatal and recovered cases of coronavirus disease 2019 (COVID-19) in Wuhan, China: a retrospective study. Chin Med J (Engl) 2020.

175. Chen T, Wu D, Chen H, Yan W, Yang D, Chen G, Ma K, Xu D, Yu H, Wang H et al: Clinical characteristics of 113 deceased patients with coronavirus disease 2019: retrospective study. BMJ (Clinical research ed) 2020, 368:m1091-m1091.

176. Chen L, Liu HG, Liu W, Liu J, Liu K, Shang J, Deng Y, Wei S: Analysis of clinical features of 29 patients with 2019 novel coronavirus pneumonia. Zhonghua jie he he hu xi za zhi = Zhonghua jiehe he huxi zazhi = Chinese journal of tuberculosis and respiratory diseases 2020, 43(3):203-208.

177. Chen J, Qi T, Liu L, Ling Y, Qian Z, Li T, Li F, Xu Q, Zhang Y, Xu S et al: Clinical progression of patients with CoVID-19 in Shanghai, China. The Journal of infection 2020.

178. Chang D, Lin M, Wei L, Xie L, Zhu G, Dela Cruz CS, Sharma L: Epidemiologic and Clinical Characteristics of Novel Coronavirus Infections Involving 13 Patients Outside Wuhan, China. JAMA 2020, 323(11):1092-1093.

179. Chen G, Wu D, Guo W, Cao Y, Huang D, Wang H, Wang T, Zhang X, Chen H, Yu H et al: Clinical and immunologic features in severe and moderate Coronavirus Disease 2019. The Journal of clinical investigation 2020.

180. Liu W, Tao ZW, Lei W, Ming-Li Y, Kui L, Ling Z, Shuang W, Yan D, Jing L, Liu HG et al: Analysis of factors associated with disease outcomes in hospitalized patients with 2019 novel coronavirus disease. Chin Med J (Engl) 2020.

181. Feng Y, Ling Y, Bai T, Xie Y, Huang J, Li J, Xiong W, Yang D, Chen R, Lu F et al: COVID-19 with Different Severity: A Multi-center Study of Clinical Features. Am J Respir Crit Care Med 2020.

182. Grasselli G, Zangrillo A, Zanella A, Antonelli M, Cabrini L, Castelli A, Cereda D, Coluccello A, Foti G, Fumagalli R et al: Baseline Characteristics and Outcomes of 1591 Patients Infected With SARS-CoV-2 Admitted to ICUs of the Lombardy Region, Italy. Jama 2020.

\section{Figures}

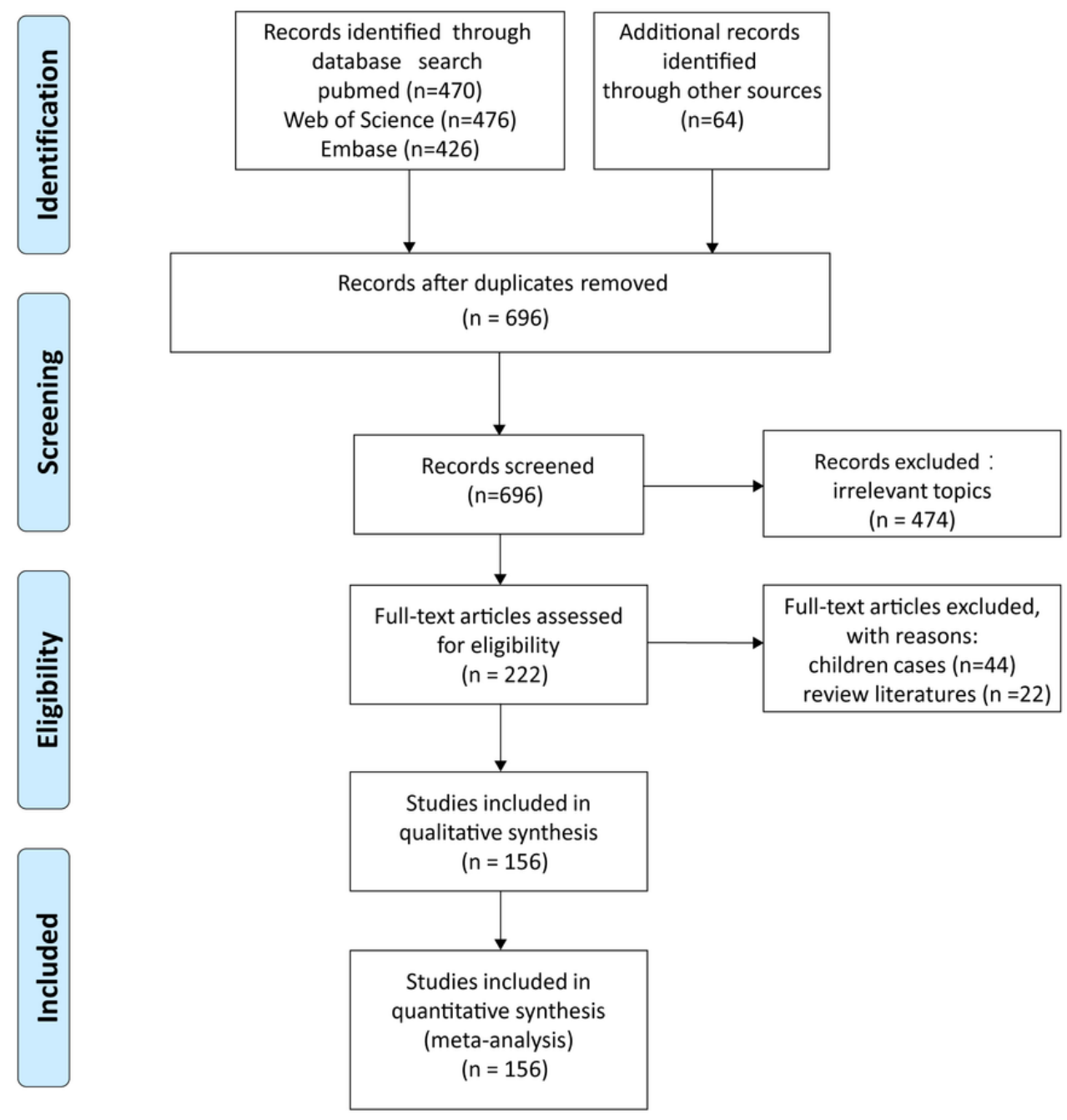

Figure 1

A flow diagram of the inclusion criteria of studies eligible for meta-analysis. 


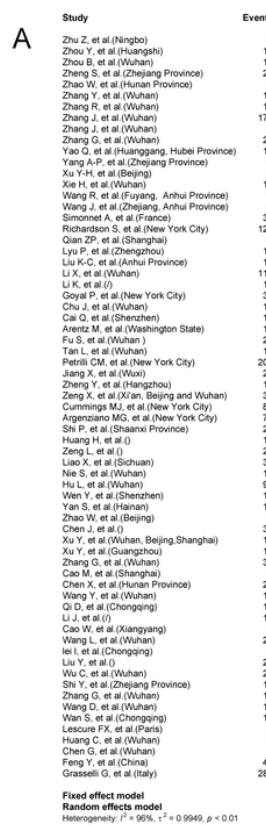

C
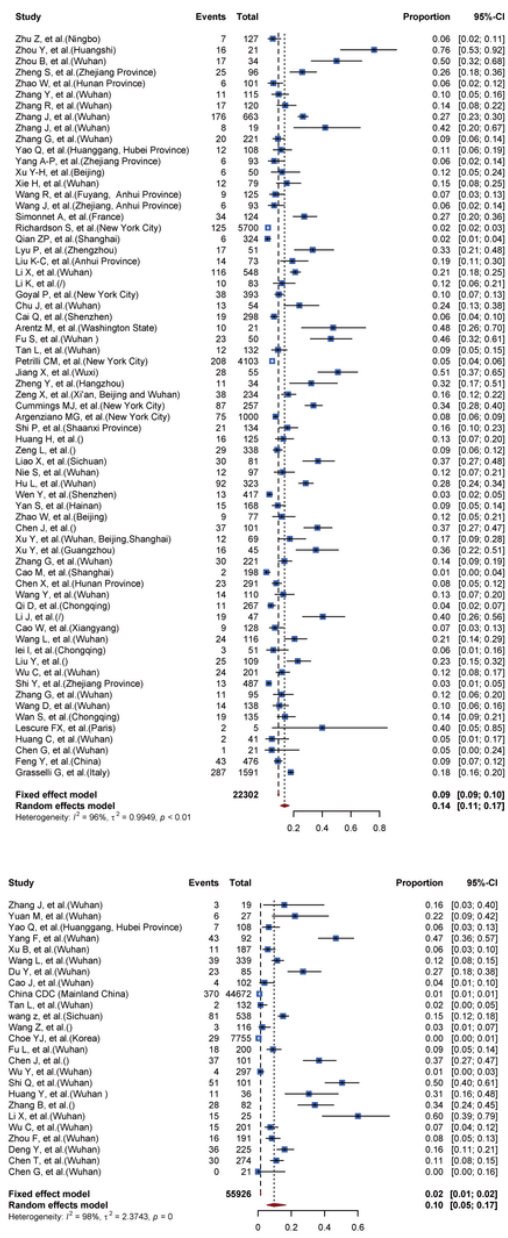

B

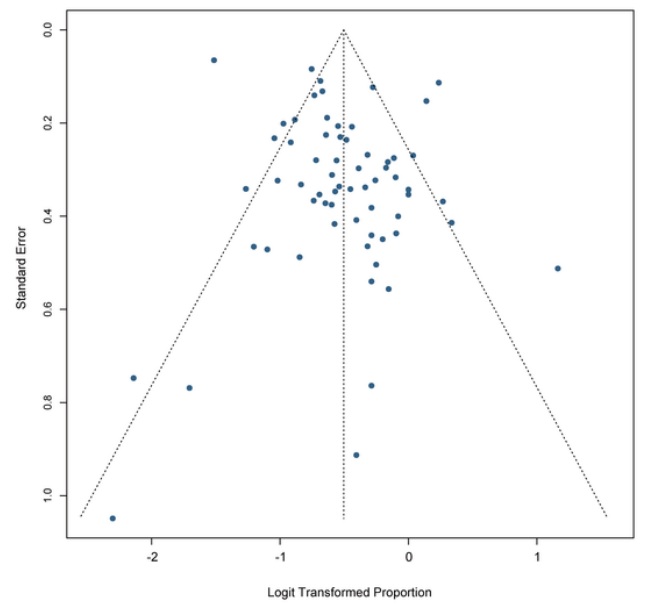

D

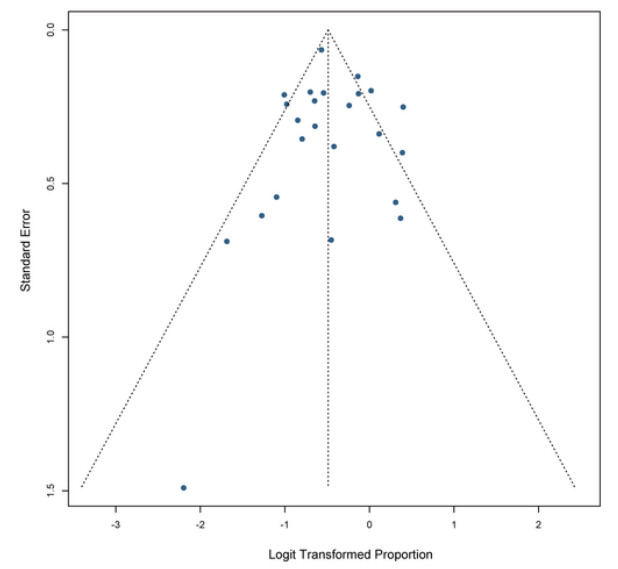

\section{Figure 2}

Forest plot and funnel plot of the proportion of female admitted into ICU or with severe/critical illness (Fig 2-A, Fig 2-B); Forest plot and funnel plot of the proportion of female death (Fig 2-C, Fig 2-D). 

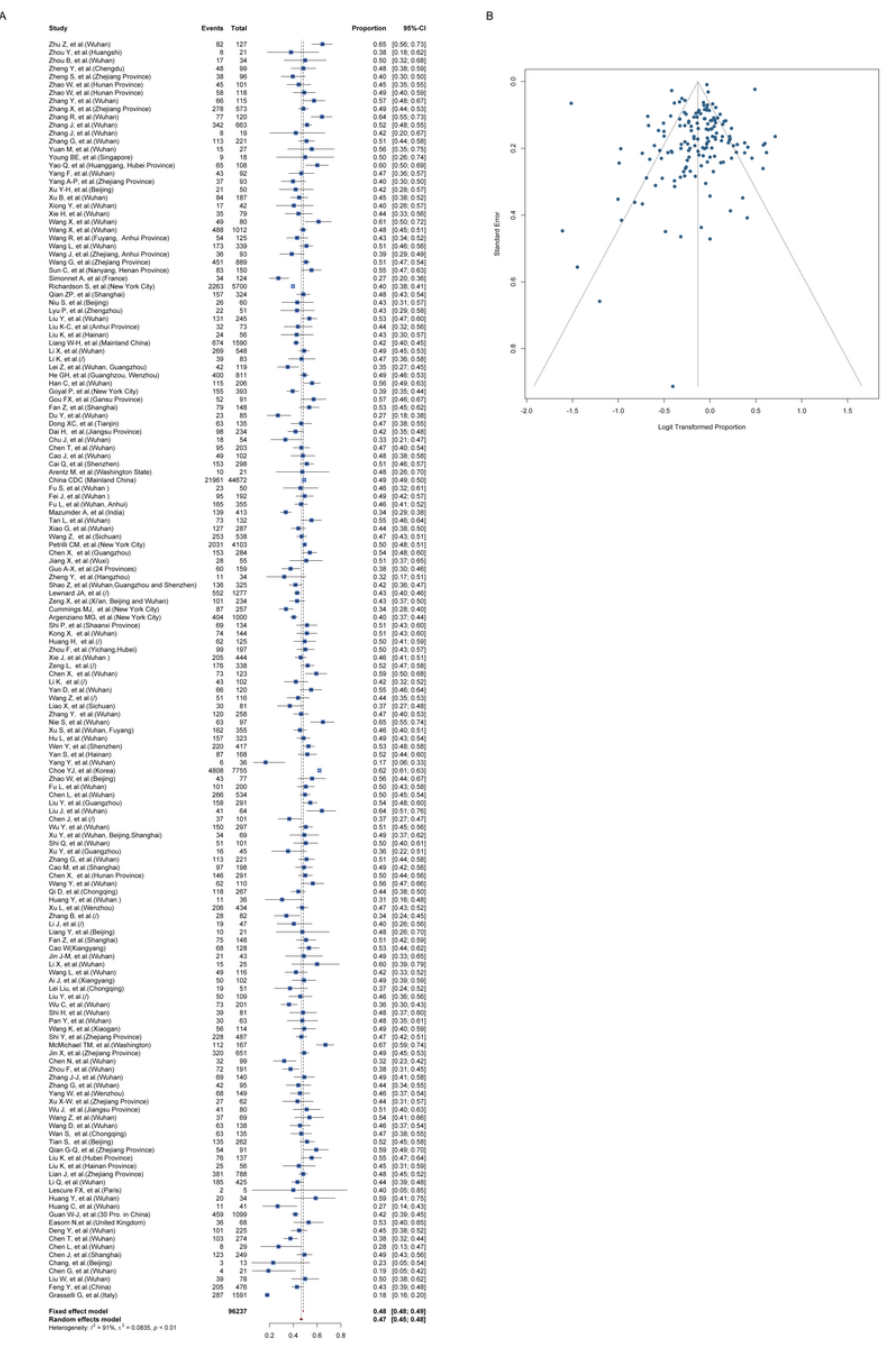

\section{Figure 3}

Forest plot and funnel plot of the proportion of female admitted into ICU or with severe/critical illness (Fig 2S-A, Fig 2S-B) 
A

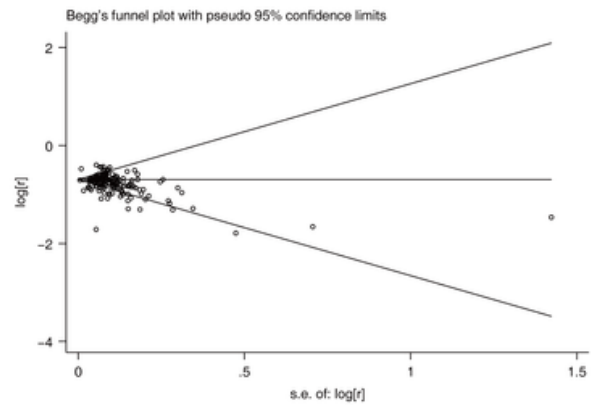

D

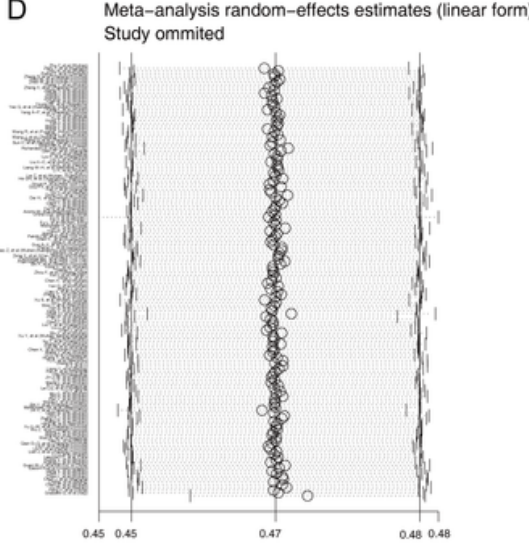

B

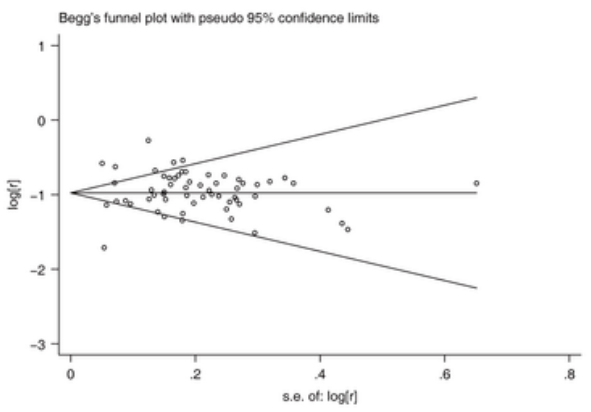

E

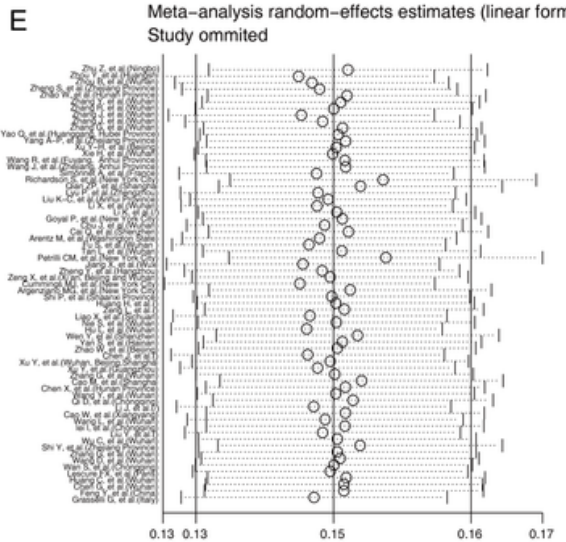

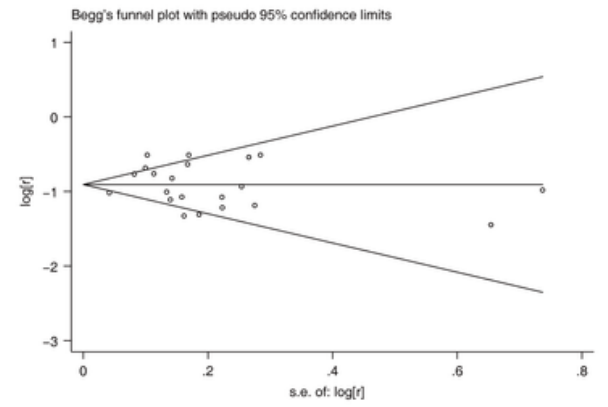

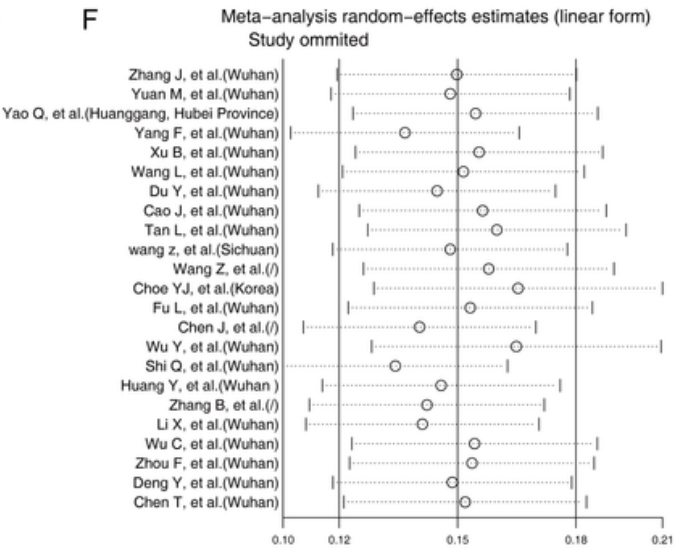

Figure 4

The publication bias plot of Begg's funnel plots and sensitivity analysis. A. Begg's funnel plots to test for the publication bias in the overall analysis of proportion of female patients; B. Begg's funnel plots to test for the publication bias in the female admitted into ICU or with severe/critical illness; C. Begg's funnel plots to test for the publication bias in the proportion of female death; D. Sensitivity analysis of the effect of individual studies in the proportion of female patients; E. Sensitivity analysis of the effect of individual studies in the proportion of female patients admitted into ICU or with severe/critical illness; F. Sensitivity analysis of the effect of individual studies in the fatality rate of female patients.

\section{Supplementary Files}

This is a list of supplementary files associated with this preprint. Click to download.

- PRISMA2009checklist.doc 